\title{
Sensory Responses during Sleep in Primate Primary and Secondary Auditory Cortex
}

\author{
Elias B. Issa and Xiaoqin Wang \\ Laboratory of Auditory Neurophysiology, Department of Biomedical Engineering, Johns Hopkins University School of Medicine, Baltimore, Maryland 21025
}

\begin{abstract}
Most sensory stimuli do not reach conscious perception during sleep. It has been thought that the thalamus prevents the relay of sensory information to cortex during sleep, but the consequences for cortical responses to sensory signals in this physiological state remain unclear. We recorded from two auditory cortical areas downstream of the thalamus in naturally sleeping marmoset monkeys. Single neurons in primary auditory cortex either increased or decreased their responses during sleep compared with wakefulness. In lateral belt, a secondary auditory cortical area, the response modulation was also bidirectional and showed no clear systematic depressive effect of sleep. When averaged across neurons, sound-evoked activity in these two auditory cortical areas was surprisingly well preserved during sleep. Neural responses to acoustic stimulation were present during both slow-wave and rapid-eye movement sleep, were repeatedly observed over multiple sleep cycles, and demonstrated similar discharge patterns to the responses recorded during wakefulness in the same neuron. Our results suggest that the thalamus is not as effective a gate for the flow of sensory information as previously thought. At the cortical stage, a novel pattern of activation/deactivation appears across neurons. Because the neural signal reaches as far as secondary auditory cortex, this leaves open the possibility of altered sensory processing of auditory information during sleep.
\end{abstract}

Key words: auditory cortex; lateral belt; sleep; primate; sensory; hearing

\section{Introduction}

The effects of arousal, attention, and anesthetics have been well documented in a variety of sensory brain areas and animal species (Hubel et al., 1959; Desimone and Duncan, 1995; Ter-Mikaelian et al., 2007; Greenberg et al., 2008). However, the role of sleep in sensory processing at the neural level has not been thoroughly investigated except for a handful of studies (see Hennevin et al. 2007 for review). Perhaps this is because it has been suggested that the brain is shut off from the external world during sleep (Steriade, 2003a) and that activity in cortex during sleep is mainly internal (Braun et al.,1997), resulting from slow oscillations or other endogenous rhythms (Steriade, 2003b). Studies of single neurons in the somatosensory (Mariotti et al., 1989), visual (Mukhametov and Rizzolatti, 1970; Coenen and Vendrik, 1972; Livingstone and Hubel, 1981; Hirsch et al., 1983), and auditory (Edeline et al., 2000) thalamus have supported this notion, leading to the proposal that the thalamus serves as a gate of sensory information into the brain. In line with this hypothesis, studies of primary visual cortex (Evarts, 1963, Livingstone and Hubel 1981) and primary somatosensory cortex (Gücer, 1979) found that al-

\footnotetext{
Received July 2, 2008; revised 0ct. 24, 2008; accepted Nov. 14, 2008.

This work was supported by National Institutes of Health Grant R01 DC-03180 (X.W.) and a Whitaker Fellowship (E.B.I.). We thank A. Pistorio, E. Bartlett, D. Bendor, P. Crum, and Y. Zhou for help with animal care; Y. Zhou for A1 data used in the frequency map of one animal; and P. Crum and T. Hackett for histological reconstruction of the LB recording site.

Correspondence should be addressed to Xiaogin Wang, Laboratory of Auditory Neurophysiology, Department of Biomedical Engineering, Johns Hopkins University School of Medicine, 720 Rutland Avenue, Traylor 410, Baltimore, MD 21205. E-mail: xiaoqin.wang@jhu.edu.

DOI:10.1523/JNEUROSCI.3086-08.2008

Copyright $\odot 2008$ Society for Neuroscience $\quad$ 0270-6474/08/2814467-14\$15.00/0
}

most all cortical neurons responded more weakly during sleep than during wakefulness.

In the auditory system, however, it is not clear to what extent primary auditory cortex (A1) is responsive to sounds during sleep. Early studies of single neurons found that activity was weaker during sleep than during wakefulness (Murata and Kameda, 1963; Brugge and Merzenich, 1973), but two recent studies recording from larger samples of neurons found weakly depressed population activity in sleep and even found some neurons that responded more strongly in sleep than in wakefulness (Peña et al., 1999; Edeline et al., 2001). These findings were bolstered by a PET imaging study showing that auditory cortex was identically activated in sleep and wakefulness by simple (tone) and complex (subject's name) sounds (Portas et al., 2000). Other imaging studies, however, found that activation in the auditory regions of the temporal lobe decreased during sleep (Czisch et al., $2002,2004)$. Given these conflicting results, the prevailing model of a thalamus-gated auditory cortex may be too simplified. From a functional standpoint, it has been shown that sleeping subjects can still tell difference between their name and those of others (Oswald et al., 1960), and evoked potential studies in humans have suggested that the signal, although altered, still carries information as to semantic meaning (Bastuji et al., 2002).

In the present study, we investigated neural responses to sounds in auditory cortex of naturally sleeping marmoset monkeys (Callithrix jacchus). Recordings were performed in A1 and a secondary auditory area, lateral belt (LB). Data from LB were compared with those from $\mathrm{A} 1$ to extend on previous work in thalamus and A1. We found that responses were only slightly $(<10 \%)$ weaker on average during sleep than during the awake condition in both cortical areas. Interestingly, modulation was 
heterogeneous across neurons such that responses in sleep could be enhanced, suppressed, or unchanged compared with wakefulness. This represents a significant departure from the model of a simple thalamic gate, and these results suggest the need for further study to understand the mechanisms that give rise to the complex effects of sleep in auditory cortex.

\section{Materials and Methods}

Subjects and surgery. We used a chronic recording preparation developed in our laboratory for marmoset monkeys which allows a lateral approach to auditory cortex. A surgery was performed to implant two head posts that were used to fix the animal's head during subsequent experiments. Before implant surgery, subjects were adapted to sit quietly in a primate restraint chair. Details of the implant surgery and recovery are detailed previously (Lu et al., 2001). After recovery from surgery, daytime recording sessions ensued. After at least a month of daytime recording, a flat-tipped $1 \mathrm{~mm}$ stainless steel screw was implanted into the caudal aspect of the temporal lobe for measuring the electroencephalogram (EEG). The signal was referenced to a ground screw located above frontal cortex. After this procedure, animals were switched to night recordings. Within a week, animals became comfortable sleeping in an upright position with their head fixed, and they adapted to sleeping in the chamber even while sounds were playing. All experimental procedures were approved by the Johns Hopkins University Animal Use and Care Committee.

Physiological recordings. The EEG signal was bandpass filtered (1-500 $\mathrm{Hz})$ and amplified $(10,000 \times)$ using an A-M Systems 1800 differential amplifier. The microelectrode signal was two-stage filtered using a Grass P15 preamplifier $(300 \mathrm{~Hz}-10 \mathrm{kHz}, 100 \times)$ and a Tucker-Davis Technologies PC1 amplifier $(300 \mathrm{~Hz}-7 \mathrm{kHz}, 100 \times)$. Both signals were monitored online on a two channel digital oscilloscope (Hameg) and digitized for later use. Extracellular action potentials were recorded using 2-4 M $\Omega$ tungsten microelectrodes (A-M Systems), and single units were identified by sorting spike waveforms online using a template-matching algorithm (Wörgötter et al., 1986) (MSD, Alpha-Omega Engineering). The quality of spike waveforms was continuously monitored online by the experimenter throughout the testing period of each unit. Testing of the unit would end if spike SNR dropped below $\sim 15 \mathrm{~dB}$ or if the spike waveform could no longer be clearly identified as a single-unit.

A hydraulic microdrive (Trent-Wells) was used to advance the electrode slowly forward stopping every 50 micrometers for at least a few minutes. Units were searched for solely based on their spontaneous firing, and we did not pass on any units encountered. As a result, units were generally recorded in close proximity to the first spike detected (median distance $=260 \mu \mathrm{m}$ ). However, the precise laminar location of each unit could not be specified especially for comparison between sessions because our reference (the first spike encountered) was an unreliable marker of the surface. First spike depth depended on thickness of dura, variations in granulation tissue, proximity to the curvature of the sulcus, and orthogonality of the electrode penetration to the cortical surface. Regardless of these factors, a majority of our recordings were clustered toward the surface and were likely from the supragranular layers (75th percentile $=440 \mu \mathrm{m}$ from first spike).

Identification of $A 1$ and $L B$. Primary auditory cortex was identified based on its proximity to the lateral sulcus (LS, marked during implant surgery), responsiveness to pure tones, and presence of a clear rostral-tocaudal (low-to-high) tonotopic gradient. After identifying A1, we recorded from LB by moving further lateral ( $\sim 4 \mathrm{~mm}$ away from the LS) (Kaas and Hackett, 2000). LB neurons often responded more strongly to noise stimuli than to tones (Rauschecker et al., 1995) and were poorly frequency-tuned to tones if at all. We caution here that although our recordings were from middle LB as opposed to anterior or caudal LB (Kaas and Hackett, 2000), no effort was made to distinguish LB from
B

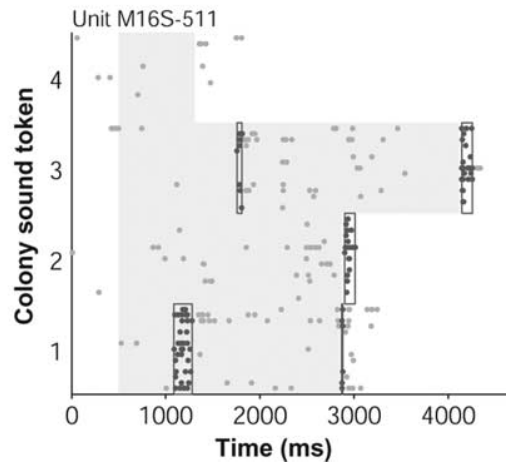

parabelt because no physiological criteria currently exist to differentiate these two fields. Recordings may well have encroached on parabelt judging by distance from LS and postmortem histological examination of lesions placed near the recording sites in one animal (second animal is still in use).

Classification of behavioral state. Marmosets are diurnal and sleep at night, so recordings were performed from the hours of 8:00 P.M. to 4:00 A.M. Animals naturally slept, passing through 5-10 sleep cycles depending on duration of the experiment. In two of the animals, the chamber lights were left on while they slept. The two other animals slept in the dark. Both EEG (divided into 4 frequency bands: $\delta=0.7-4.2, \theta=$ $4.2-7.5, \alpha=7.5-12$, and $\beta=12-20$ ) and video monitoring (sensitive in the infrared range) were used to assess an animal's behavioral state. Three major behavioral states were readily identified using standard human sleep scoring techniques except that video monitoring was used in lieu of the EMG for identifying loss of muscle tone in REM (Carskadon and Rechtschaffen, 2000): Awake-eye openings, facial movements observable on video monitor leading to myogenic potentials in the EEG, and energy in all EEG frequency bands including the $\beta$ band; SWS-increased amplitude and prevalence of low-frequency activity in the $\delta$ range and decreased energy in the $\beta$ band; and REM-low amplitude, high frequency ( $\alpha$ and $\beta$ ) EEG and twitching of face muscles observable on video monitor with no myogenic potentials on EEG trace (see supplemental Fig. S1, available at www.jneurosci.org as supplemental material, for comparison of EEG frequency spectra in awake, SWS, and REM). In two of the animals, REM onset was identified by the tail uncurling and dropping, and REM offset coincided with lifting of the tail back into a tucked position, presumably from recovery of muscle tone. In general, we used conservative criteria because we required clear and stable behavioral states for measuring neural responses. Both EEG and video monitoring criteria had to be unambiguously satisfied and, to limit variability, data from transitions into or out of awake, SWS, and REM or from light periods of SWS (i.e., early stage 3 ) were not considered. In a few instances, we manually awakened the animal by playing loud sounds, turning the lights on, and entering the chamber to increase awake arousal.

Acoustic stimuli. Acoustic stimuli included pure tones, bandpass noise, sinusoidal amplitude modulated tones, Gaussian or rectangular click trains, and vocalizations. Not all stimuli were played for each neuron. Rather, specific stimuli that drove the neuron in question were chosen and on average 6 effective stimuli were tested per neuron. For the majority of our analyses, we pooled data across all stimuli tested because minor differences were observed between different stimulus types. We used the data for all stimuli that elicited a significant driven response ( $>4$ SEM above spontaneous rate) in at least one of the behavioral conditions (awake, SWS, or REM). All stimuli were delivered in free-field from a speaker (B\&W 601) located $0.9 \mathrm{~m}$ directly in front of the animal. Generally, stimuli were 200 or $500 \mathrm{~ms}$ in duration which was sufficient to elicit sustained responses in addition to onsets. So as to limit sleep disruption, stimuli were not made too long (except for vocalizations which could be 


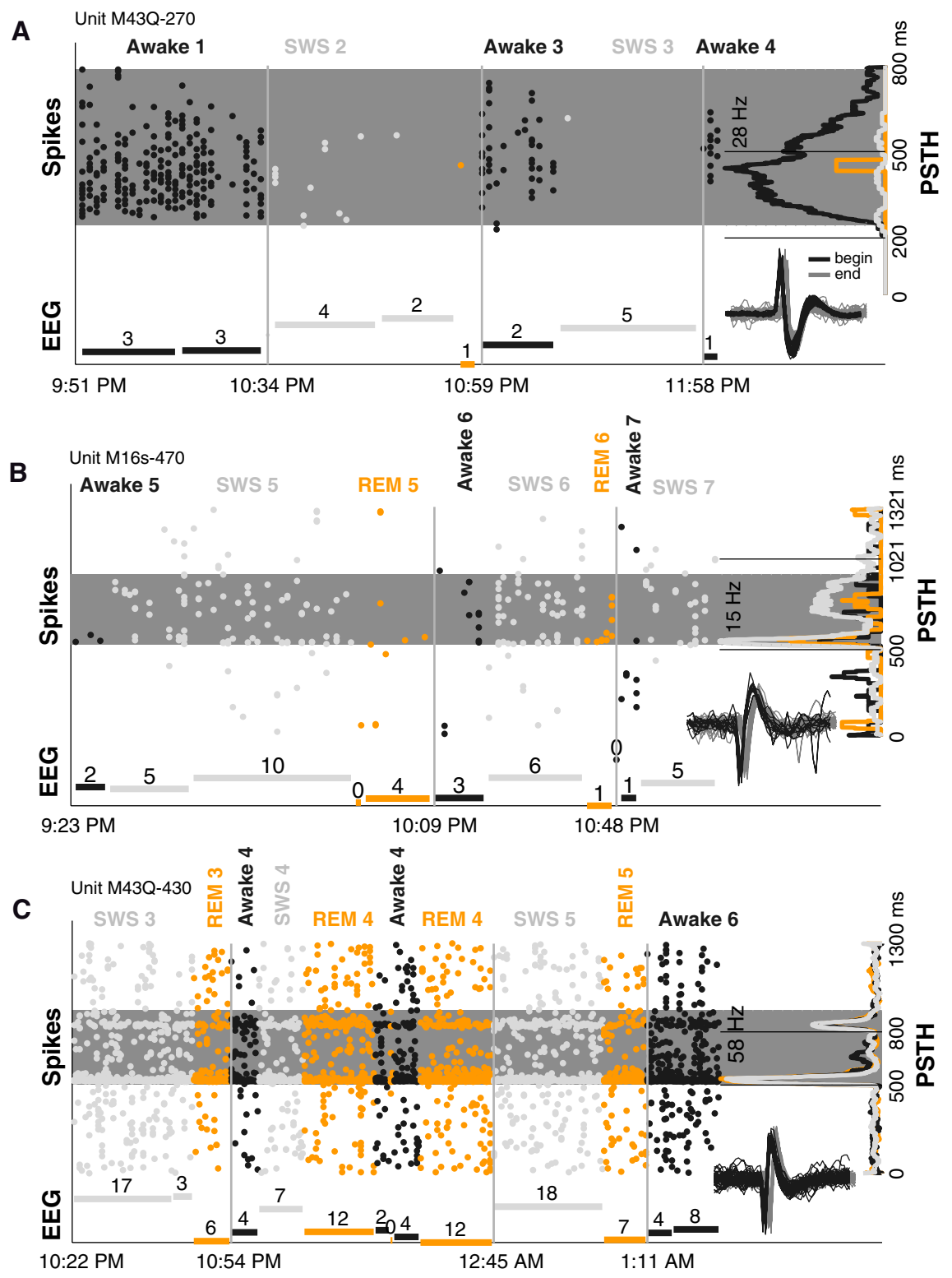

Figure 2. Example units modulated during sleep. $\boldsymbol{A}$, Spiking activity and EEG measured during different time points while the animal slept (data were not collected continuously). This unit's response to a sAM tone (carrier frequency $=8.3 \mathrm{kHz}$, modulation frequency $=256 \mathrm{~Hz}$, sound level $=30 \mathrm{dBSPL}$ ) consistently went down when the unit was tested in sleep (gain $=-96 \%$ in SWS) (dark gray, awake; light gray, SWS; and orange, REM). The spike raster (oriented vertically, each column represents a single trial) shows a clear loss of spiking on entry into SWS in episode 2, a recovery of response on returning to awake in episode 3, and a loss of response again in SWS of episode 4. Far right, The PSTH averages the activity across all trials for each state and is plotted on its side. The clear response in awake is absent in SWS and REM. Gray shaded strip is the analysis window determined by our windowing algorithm. Dashed lines demarcate stimulus playing. Bottom, The normalized EEG amplitude (minimum subtracted) for each epoch tested is shown (times each episode began are marked on abscissa; breaks between EEG bars within an episode denote noncontinuous measurements; and numbers above each bar denote duration of measurement in minutes). When driven spiking is high in awake periods, EEG amplitude is low. In SWS periods, high EEG amplitude accompanies diminished firing. Inset, first 50 (black) and last 50 (gray) spike waveforms collected of the unit during the 2.5-h-long session. Horizontal offset is for display purposes only. $\boldsymbol{B}$, Same format as $\boldsymbol{A}$. This unit's response to a marmoset vocalization sound (type $=$ tsik, sound level $=80$ $\mathrm{dBSPL}$ ) went up in SWS (gain $=86 \%$ in SWS) but not in REM. Firing rate increased when EEG amplitude (SWS) increased. Session spanned $2 \mathrm{~h}$. C, Same format as $\boldsymbol{A}$. Unit's response to a tone (frequency $=8.7 \mathrm{kHz}$, sound level $=60 \mathrm{~dB}$ ) was similar in awake, SWS, and REM (gain $=-11 \%$ in SWS). Regardless of multiple large changes in EEG amplitude and behavioral state over the 3-h-long session, the unit remained consistently well driven by the stimulus, and the PSTH retained its distinct onset and offset pattern in all three states.

a few seconds long), and presentation rates were generally kept low (0.1-1 Hz). Interstimulus intervals were at least twice the duration of the stimulus and often much longer to avoid neural adaptation. Experiments were performed in a double-walled sound isolation chamber (Industrial
Acoustics) lined with 3 inch acoustic absorption foam (Sonex) to create a soundproof environment.

Data analysis. We developed an algorithm similar to that of Legéndy and Salcman (1985) for detecting defined periods of driven activity (Hanes et al., 1995; Sheinberg and Logothetis, 2001). The algorithm starts with a $30 \mathrm{~ms}$ seed window (1.5× SEM above spontaneous rate), centered at a local maximum, that grows in either the left or right direction in $30 \mathrm{~ms}$ segments. To determine when to stop expanding the window, the algorithm uses thresholding with hysteresis. Window segments are added as long as they meet a soft criterion $\left(p_{\text {soft }}<0.1\right)$ and the whole window meets a more stringent hard criterion $\left(p_{\text {hard }}<0.01,3 \times\right.$ SEM above spontaneous rate). Gaussian statistics were assumed for estimates of mean firing rate in a particular analysis window. The mean and SEM were computed and compared with mean spontaneous firing to determine a $p$-value. The windows turned up by the algorithm were only guides for data analysis. Final firing rates were required to be $4 \times$ SEM above spontaneous rate $(p<0.0001)$. When multiple discrete windows were detected, we used an analysis window that ran from the beginning of the first window to the end of the last window. In general, our algorithm performed well for different conditions (for low or high driven and background firing rates, for small or large numbers of trials, and for long-lasting or brief events) agreeing with what was expected from visual inspection of the responses (Fig. 1).

Using the mean discharge rate (with spontaneous rate subtracted) over the analysis window found by our algorithm, we computed percentage modulation in sleep for each stimulus by comparing firing rates from two different behavioral states according to the following formulas:

$$
\begin{aligned}
\%_{\text {Gain }} \text { SWS-Awake } & =100 * \frac{r_{\text {SWS }}-r_{\text {Awake }}}{\max \left(\left|r_{\text {SWS }}\right|,\left|r_{\text {Awake }}\right|\right)} \\
\% \text { Gain }_{\text {REM-Awake }} & =100 * \frac{r_{\text {REM }}-r_{\text {Awake }}}{\max \left(\left|r_{\text {REM }}\right|,\left|r_{\text {Awake }}\right|\right)},
\end{aligned}
$$

where $r_{\mathrm{SWS}}, r_{\mathrm{REM}}$, and $r_{\text {Awake }}$ are the discharge rates during the SWS, REM, and awake conditions, respectively.

The unit's overall gain was taken as the mean of all the individual stimulus gains. Conclusions were unaffected when stimulus gains were used instead of averaged unit gains. A unit was considered significantly modulated if sleep firing rate differed by $>3 \times$ SEM from awake firing rate for any stimulus $(p<0.01)$. We did not correct for multiple comparisons because the number of stimuli tested for each unit was not large (mean $=6$ ), and we were already using a strict significance criterion $(>3 \times$ SEM). Usually, the number of stimuli traded off with the number of trials that could be run for each stimulus so that there was less of an advantage to testing multiple stimuli. SDs of gain values were estimated using the jackknife resampling technique (Efron, 1982).

To perform an unbiased nonparametric three-way comparison between awake, SWS, and REM, we ordered firing rates in the three states 
for each unit. We then counted the frequency of certain patterns. If REM and SWS were correlated, then the pattern XXA or AXX (where X = REM or SWS) should occur more often than chance, and the pattern XAX where SWS and REM show changes in opposite directions should occur less often than chance (chance levels: $\left.p_{\mathrm{XAX}}=1 / 3, p_{\mathrm{XXA} \mid \mathrm{AXX}}=2 / 3\right)$.

Using the response windows detected by our algorithm, responses were considered onsets if they began in the first $60 \mathrm{~ms}$ of the response regardless of response latency and lasted $<110$ ms. Responses continuing past $110 \mathrm{~ms}$ were counted as sustained responses, and offset responses began and ended after the stimulus ended.

For correlating poststimulus time histograms (PSTHs), we first subtracted spontaneous rate, smoothed PSTHs by a $20 \mathrm{~ms}$ moving average filter, and performed a dot product according to the following:

$$
\begin{aligned}
& \rho_{\text {SWS,Awake }}= \\
& \frac{\left(\mathrm{PSTH}_{\text {Awake }}-\mu_{\text {Awake }}\right) \bullet\left(\mathrm{PSTH}_{\mathrm{SWS}}-\mu_{\mathrm{SWS}}\right)}{\left|\mathrm{PSTH}_{\text {Awake }}-\mu_{\text {Awake }}\right| \mathrm{PSTH}_{\mathrm{SWS}}-\mu_{\mathrm{SWS}} \mid}
\end{aligned}
$$

$\rho_{\text {REM,Awake }}=$

$$
\frac{\left(\mathrm{PSTH}_{\text {Awake }}-\mu_{\text {Awake }}\right) \bullet\left(\mathrm{PSTH}_{\mathrm{REM}}-\mu_{\mathrm{REM}}\right)}{\left|\mathrm{PSTH}_{\text {Awake }}-\mu_{\text {Awake }}\right|\left|\mathrm{PSTH}_{\mathrm{REM}}-\mu_{\mathrm{REM}}\right|},
$$

where $\mathrm{PSTH}_{\text {Awake, }}, \mathrm{PSTH}_{\mathrm{SWS}}$, and $\mathrm{PSTH}_{\mathrm{REM}}$ are the PSTHs taken from stimulus onset to $300 \mathrm{~ms}$ after stimulus offset during the awake, SWS, and REM conditions, respectively.

For bursting analysis, interspike intervals (ISIs) were computed during stimulus presentation across all stimuli tested. Percentage bursting was the number of ISIs $<x$ (where $x=$ $4,5,8$, or $10 \mathrm{~ms}$ ) divided by the total number of ISIs (Edeline et al., 2001).

The vector strength (VS) was computed to determine synchronization to the cycles of periodic stimuli as follows:

$\mathrm{VS}=$

$$
\frac{1}{n} \sqrt{\left(\sum_{i} \cos \left(2 \pi t_{i} / T\right)\right)^{2}+\left(\sum_{i} \sin \left(2 \pi t_{i} / T\right)\right)^{2}},
$$

where $t_{i}$ is the time of the $i$ th of $n$ spikes and $T$ is the period of the modulation equivalent to the interclick interval of a click train (Goldberg and Brown, 1969). Stimulus synchronization was considered significant if the Rayleigh statistic ( $\mathrm{RS}=2^{\star} n_{\text {spikes }}{ }^{*} \mathrm{VS}^{2}$ ) was $>13.8$ (Mardia and Jupp, 2000).

The similarity of two tuning curves was measured using a correlation coefficient as follows:

$$
\begin{gathered}
\rho_{\text {SWS,Awake }}=\frac{\left(r_{\text {Awake }}-\mu_{\text {Awake }}\right) \bullet\left(r_{\text {SWS }}-\mu_{\text {SWS }}\right)}{\left|r_{\text {Awake }}-\mu_{\text {Awake }}\right|\left|r_{\text {SWS }}-\mu_{\text {SWS }}\right|} \\
\rho_{\text {REM,Awake }}=\frac{\left(r_{\text {Awake }}-\mu_{\text {Awake }}\right) \bullet\left(r_{\text {REM }}-\mu_{\text {REM }}\right)}{\left|r_{\text {Awake }}-\mu_{\text {Awake }}\right|\left|r_{\text {REM }}-\mu_{\text {REM }}\right|},
\end{gathered}
$$

where $r$ is the driven rate vector (spontaneous rate subtracted) in each state (awake, SWS, or REM) ordered by stimulus number, and $\mu$ is the mean driven rate in each state across all stimuli.

Only units that had a significant response in both behavioral states
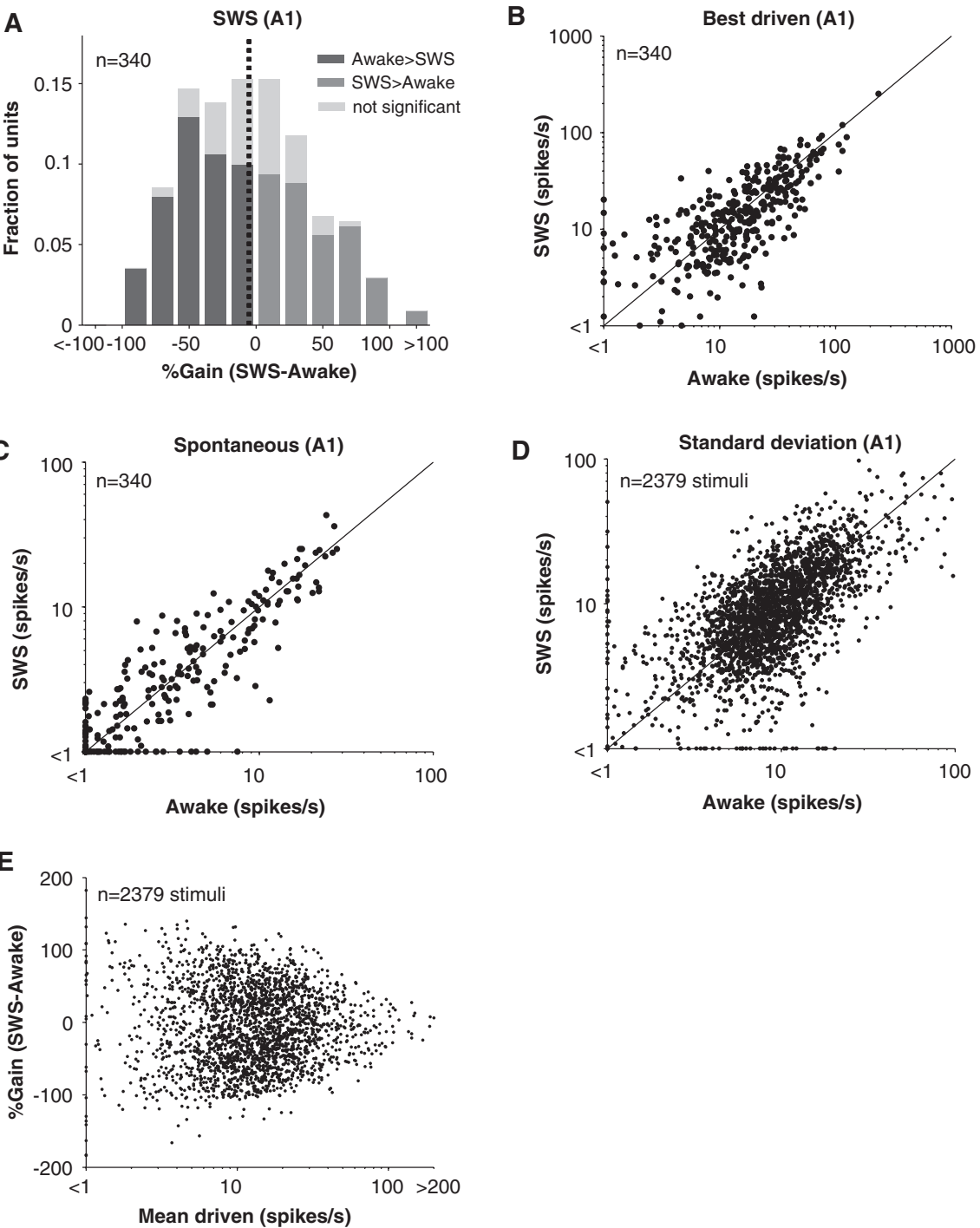

Figure 3. Stimulus-driven and spontaneous activity in A1 during awake and SWS. A, Histogram of \%Gain (SWS-awake) for whose responses went up in SWS. Lightly shaded portions of bars represent responses that did not significantly differ between awake and SWS ( $<3 \times$ SEM difference). Mean gain $=-5 \%$ (vertical dotted line). $\boldsymbol{B}$, Scatter plot of stimulus-evoked firing rates 340). C, Scatter plot of unit spontaneous firing rates in awake and SWS. Spontaneous rates did not significantly differ ( $p=$ Wicoxon rank sum, $n=340)$. D, Scatter plot of the SD of firing rates in awake and SWS. SD of driven responses did not significantly differ ( $p=0.90$, Wilcoxon rank sum, $n=2379$ ). $E$, \%Gain as a function of driven rate for all stimuli tested in A1. No trend is present with driven rate $\left(r^{2}=2.2^{*} 10^{-5}, p=0.82, n=2379\right)$.

were considered for the PSTH similarity, vector strength, and tuning similarity analyses. Wherever two distributions were statistically compared throughout the text and figures, a Wilcoxon rank sum test was used, and the correlation between two variables was quantified using Pearson's product-moment correlation coefficient.

To classify cells as putative fast spiking (inhibitory) or regular spiking (excitatory), the width of the spike waveform was used (Barthó et al., 2004; Mitchell et al., 2007; Zoccolan et al., 2007). The waveform was divided into three segments (peak, trough, and afterhyperpolarization) based on its zero crossings about positive and negative peaks. A spike was considered narrow if trough width was $<500 \mu$ s and total spike duration was $<2.5 \mathrm{~ms}$. These cutoffs were chosen based on visual inspection of the spike width distributions and are not intended to match those in the literature. In only a subset of neurons could this analysis be performed ( $n=266$ ) because spike waveforms were not collected for all neurons. Seventeen percent $(45 / 221)$ of neurons were classified as putative inhib- 

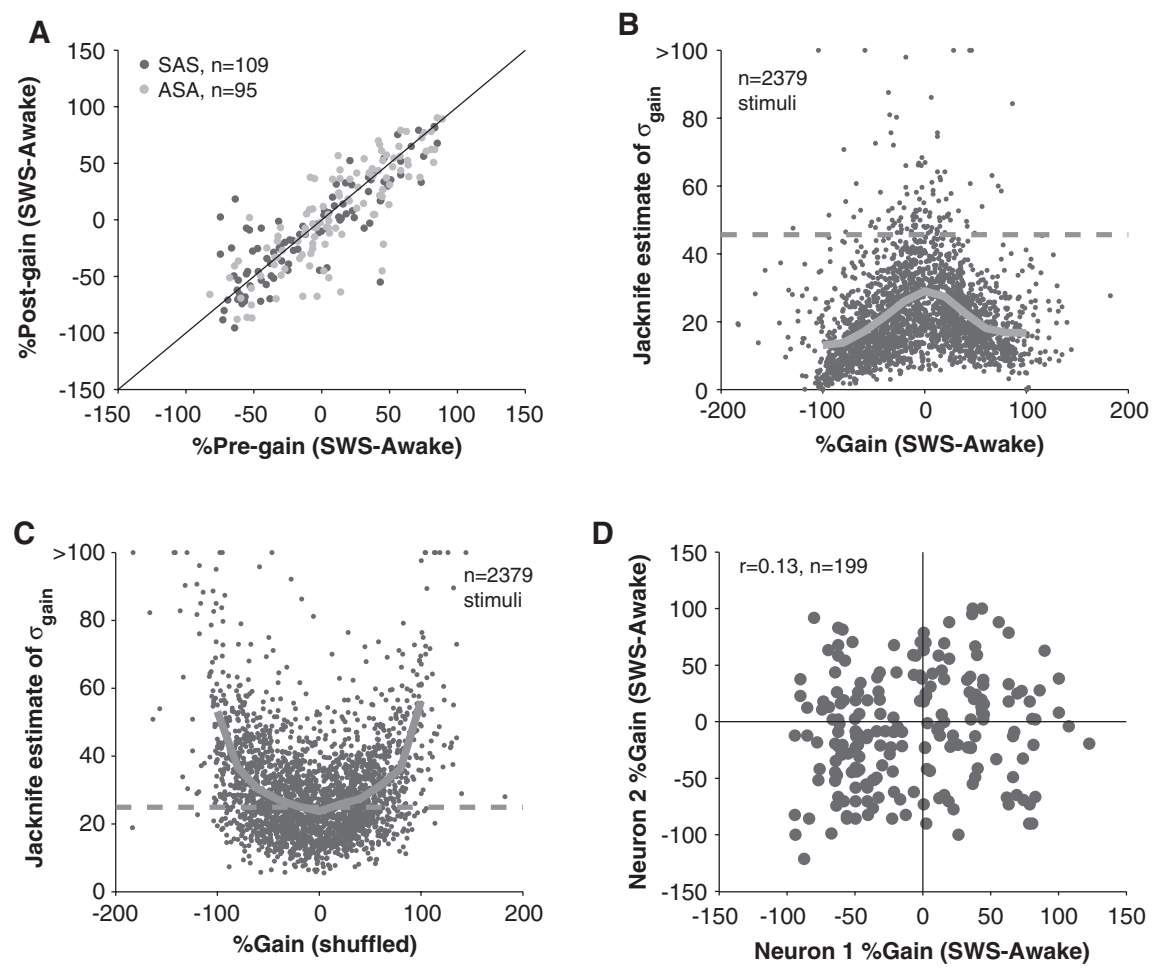

Figure 4. Reliability and precision of measured gains in SWS. $A$, In some units, a recovery control was performed. Modulation of firing was retested in a later sleep cycle. Order of testing was either awake-SWS-awake (ASA) or SWS-awake-SWS (SAS). There was a high correlation between pregains and postgains suggesting similar modulation of a neuron in two separate sleep cycles $\left(r^{2}\right.$ $=0.71, p=2.9^{*} 10^{-56}, n=203$ ). $B$, SDs of gains, estimated using the jackknife technique, were used to approximate noise arising from inherent variability in neural firing rates and behavioral state. Noise levels at each gain (solid gray line represents mean) were a decreasing function of the absolute value of gain. Especially for high gains, noise was low. The mean $46 \%$ change in firing rate observed during SWS (dashed gray line) was generally above noise levels (mean $=21 \%$ ). C, If individual trials are randomly assigned to awake or SWS, the magnitude of gain becomes positively correlated with noise in the measurement. High gains are simply the result of high variability in firing rates. This was not the trend in $\boldsymbol{B}$, suggesting a systematic effect of sleep beyond measurement noise. D, SWS modulation of neurons recorded within $200 \mu \mathrm{m}$ of each other. Gains of neighboring neurons were not correlated $\left(r^{2}=0.02, p=0.06, n=199\right)$. Often neurons were modulated in opposite directions (top left and lower right quadrant of plot) such that the response of one neuron would increase on entry to SWS (positive gain) whereas the response of a second, nearby neuron would decrease (negative gain).

itory interneurons using these criteria (supplemental Fig. S2, available at www.jneurosci.org as supplemental material).

\section{Results}

\section{SWS modulation of responses in A1}

Data were collected from a total of 497 single units, 401 units from primary auditory cortex (A1) of three animals and 96 units from lateral belt (LB) of two animals. Two unique stages of sleep, slow-wave sleep (SWS) and rapid eye movement sleep (REM), were differentiated in addition to the awake state (see Materials and Methods). Units were held an average of $1.5 \mathrm{~h}$ so that, usually, responses could be measured across at least one marmoset sleep cycle ( $\sim 50 \mathrm{~min}$, Crofts et al., 2001). Most units (384/497) were tested in both SWS and REM, but this was not always the case resulting in slightly different numbers of units tested in SWS $(\mathrm{A} 1=340, \mathrm{LB}=84)$ and $\mathrm{REM}(\mathrm{A} 1=334, \mathrm{LB}=77)$. Although we only required a minimum of 3 stimulus repetitions for units to be included in analysis, units were in practice tested for $\sim 10$ repetitions with little difference in the number of repetitions collected in each state (medians: awake $=11$, SWS $=13$, REM $=10$ ).

The three example units in Figure 2 illustrate the range of effects observed in sleep. The unit in Figure $2 \mathrm{~A}$ responded with high driven rates during awake trials (dark gray) when EEG amplitude (dark bars) was low. When EEG amplitude went up dur- ing SWS (light gray), firing rate dropped to almost zero. In other cases, we were surprised to find the opposite scenario, that firing often increased during SWS (Fig. $2 B$ ). The third unit (Fig. 2C) had a nearly identical response in awake, SWS, and REM (orange). In all 3 units, similar response modulations were recovered in later sleep episodes spanning the course of hours. Units were well isolated throughout the recordings; the signal-to-noise ratio $(>20 \mathrm{~dB})$ of the spike waveforms was similar at the beginning and end of the sessions (Fig. 2, insets). The stable spike waveforms and the consistent recovery of responses suggest that modulation of responses during sleep for these 3 units cannot be accounted for by nonspecific factors like adaptation to the stimulus over the course of the session, declining cell health, poor unit isolation, or unreliable classification of behavioral state.

Similar to these example units, strong but variable modulations were encountered across the population of units tested. Units were modulated an average of $38 \%$ in both directions during SWS (Fig. $3 A$ ). Although $45 \%$ of units decreased their response significantly when the animal fell asleep, $34 \%$ of units actually increased their response significantly when the animal fell asleep. As a result of the offsetting bidirectional effects of SWS, overall activity dropped by only $5 \%$ (Fig. $3 A$, mean of gain histogram) over the A1 population ( $n=340$ units). Distributions of best driven rates (Fig. $3 B$ ) and spontaneous rates (Fig. 3C) were not significantly different between awake and SWS $\left(p_{\text {best }}=\right.$ $0.13, p_{\text {spont }}=0.80$, Wilcoxon rank sum, $n=340)$. Interestingly, the SD of neural firing during driven periods was unchanged in SWS ( $p=0.90$, Wilcoxon rank sum, $n=2379$ stimuli) (Fig. 3D). This does not support the prediction that firing should be more variable in SWS because of the up and down influences of slow oscillations (Massimini et al., 2003; Rosanova and Timofeev, 2005). One possibility is that our stimuli were too long such that they averaged out the effects of the slow oscillation. However, given the brief duration $(200 \mathrm{~ms})$ of the majority of our stimuli relative to a period of the slow oscillation (500-2000 ms), this seems unlikely (Massimini et al., 2003).

Modulation of responses in sleep may depend on where in the cell's operating regime measurements were made (near response threshold versus optimally driven). Responses at high firing rates, for example, may saturate leaving little room for observing sleep modulation (Reynolds and Chelazzi, 2004). In general, gain did not depend on firing rate $\left(r^{2}=2^{\star} 10^{-5}, p=0.82, n=2379\right)$; gain distributions became more variable (more extreme in magnitude) at low rates (Fig. $3 E$ ). This could be the result of operating closer to a unit's firing threshold at which small effects on membrane potential can lead to large changes in firing rate. Alternatively, this may be the result of increasingly variable estimates of firing rate at low driven rates when only a few spikes were elicited. Also, gain did not depend strongly on spontaneous 
rate $\left(r^{2}=0.01, p=2^{\star} 10^{-7}, n=2379\right)$. So both positive and negative gains were observed regardless of the spontaneous activity of neurons or how strongly they were driven, and mean gain was near $-5 \%$ over the population.

As a control, we tested the repeatability (accuracy) of our measures of SWS modulation by retesting approximately onehalf of the units in a later sleep cycle (mean separation between tested cycles $=70 \pm$ $33 \mathrm{~min}, n=203)$. Gains computed in later sleep cycles were strongly correlated with gains measured in previous cycles $\left(r^{2}=\right.$ $0.71, p=3^{\star} 10^{-56}, n=203$ ) (Fig. $4 A$ ). In addition to being accurate, gain measurements were relatively precise (Fig. $4 B$ ). In general, the strong average effects of sleep on firing rates (46\%, Fig. $4 B$, dashed line) were well above the estimated noise level in our measurements (21\%, Fig. 4B, solid line). This addresses the concern of whether the mix of up and down changes in firing rate during sleep was simply the result of measurement noise. The opposite trend was found; higher gains had lower estimation noise (Fig. $4 \mathrm{~B}$ ) in contrast to the upward trend that would be expected if increasing noise was contributing to the presence of high gain values (Fig. 4C).

We often recorded neurons in close proximity (see Materials and Methods) allowing us to test whether activations or deactivations were clustered. For neurons recorded within $200 \mu \mathrm{m}$ of each other, there was little correlation in their sleep gains $\left(r^{2}\right.$ $=0.02, p=0.06, n=199$ ) (Fig. $4 D$ ). Nearby neurons could display different (sometimes opposite) behaviors suggesting that the mixture of up and down effects of sleep on firing rates were simply the result of local differences between neurons and not the result of other factors like nonstationarity over time (Fig. $4 A$ ) or estimation noise (Fig. $4 B$ ) which were both ruled out. Also, these data, although not conclusive, suggest that there is not a laminar dependence of the effects of sleep because any clustering would have resulted in a statistically significant correlation. However, our recordings likely focused on the supragranular layers and may have missed differential clustering in certain layers, especially deeper layers. The apparent randomness of sleep modulation between neurons has been noted before (Livingstone and Hubel, 1981; Edeline et al., 2001). In a further analysis in which neurons were classified as excitatory neurons or inhibitory interneurons based on their spike waveforms (see Materials and Methods), the effects of sleep were similar between putative neuron classes (supplemental Fig. S2, available at www.jneurosci.org as supplemental material).

\section{Responses properties of $\mathrm{LB}$ neurons}

We reasoned that higher cortical areas may be more sensitive to a change in behavioral state because they can depend strongly on
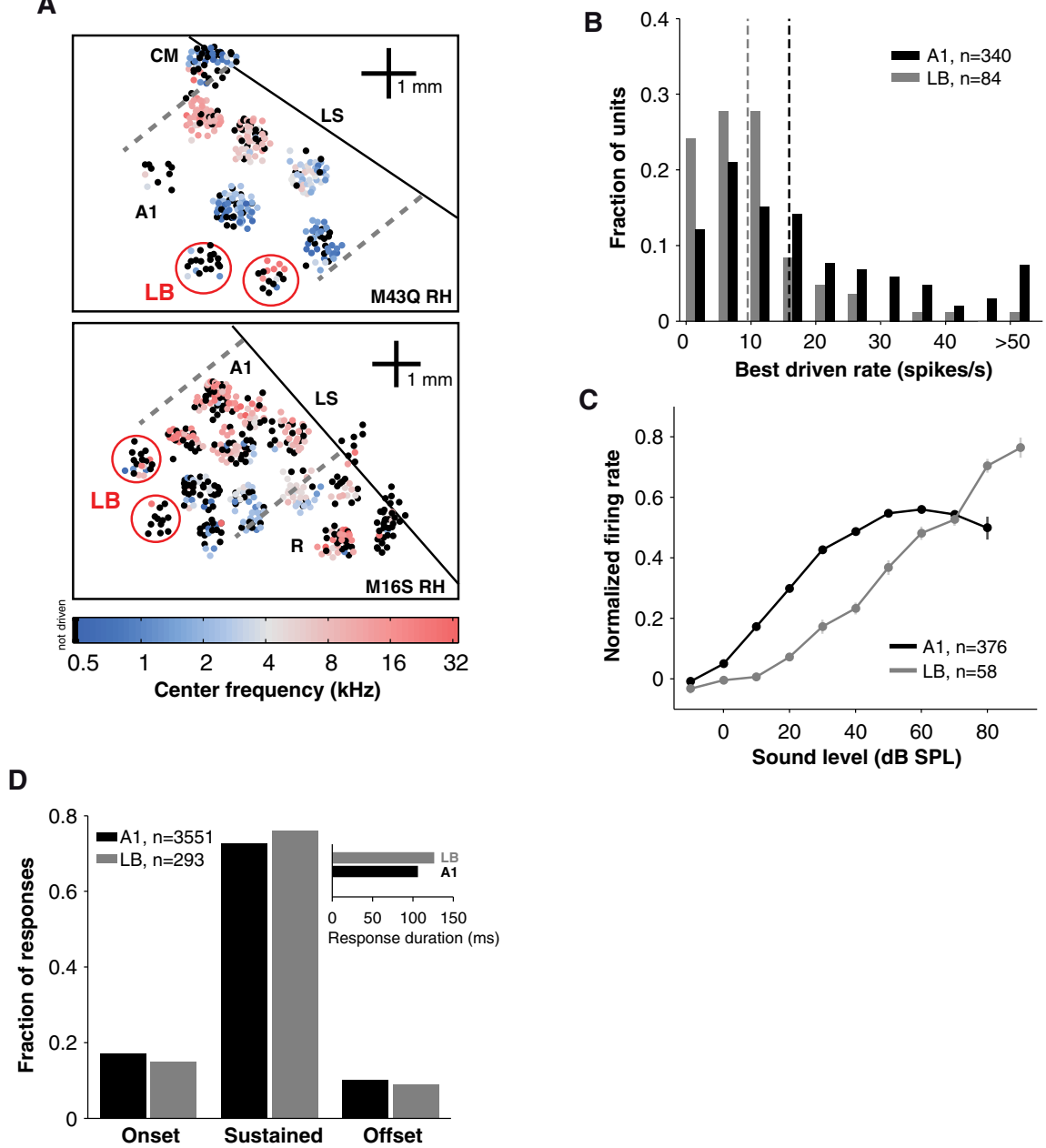

Figure 5. Recording maps and properties of lateral belt. $A, L B$ recordings were obtained in the right hemispheres of 2 animals in addition to $A 1$ recordings. The tonotopic progression in $A 1$ can be seen in the maps of both animals. In animal M43q (top), $A 1$ was bounded caudally by CM (caudomedial field). In animal M16s (bottom), A1 was bounded rostrally by R (rostral field). In both cases a clear frequency reversal is seen at the borders (dashed lines). Colored dots indicate neurons whose center frequencies were determined. The dots are slightly dispersed for display purposes. Black dots represent track locations in which center frequencies were not determined because neurons were unresponsive to tones or narrowband noise. LB recordings (red circles) were made far from the lateral sulcus (LS, diagonal black line). These regions had weak overall responses and poor tonotopic gradients as evidenced by the prevalence of black dots. $\boldsymbol{B}$, Units in LB were not driven as strongly as units in $A 1$ (median best response $L B=9.5$ rate levels in $A 1(n=376)$ and $L B(n=58)$. Curves from either awake or SWS were normalized by peak response and then windows were the dominant form of response under the awake condition. Responses lasted slightly longer in $L B$ (mean $=126$ $\mathrm{ms}$ ) than in $\mathrm{A} 1$ (mean $=106 \mathrm{~ms}$ ) (inset).

top-down processing. It is therefore important to study how sleep affects a higher auditory center especially one involved in processing more complex stimulus properties such as lateral belt (LB) (Rauschecker et al., 1995). After physiologically defining the primary area A1, we recorded from $\mathrm{LB}$, a secondary sensory area (Kaas and Hackett, 2000). Recordings were made far lateral of A1 so as to avoid the A1/belt border at which areal identity is ambiguous (Fig. 5A) (see Materials and Methods). Unlike A1 units, units in LB were hard to drive with simple stimuli and were weakly driven in general (Fig. 5B). Whereas A1 responses tapered off at high sound levels, $\mathrm{LB}$ responses continued to grow over the population (Fig. 5C). Despite lower firing rates in $\mathrm{LB}$, the proportion of sustained responses elicited was similar to A1 (Fig. $5 D)$, and mean duration of responses was actually longer in $\mathrm{LB}$ 
A

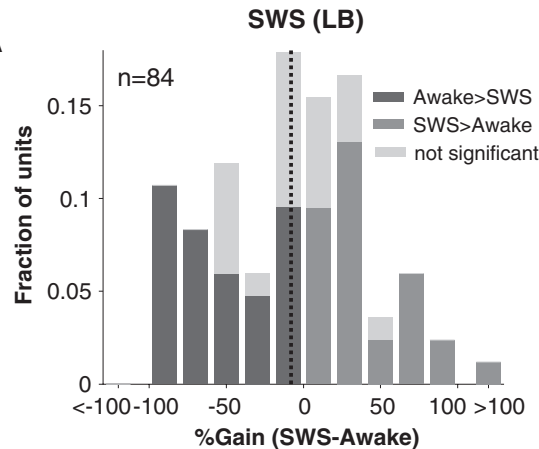

C

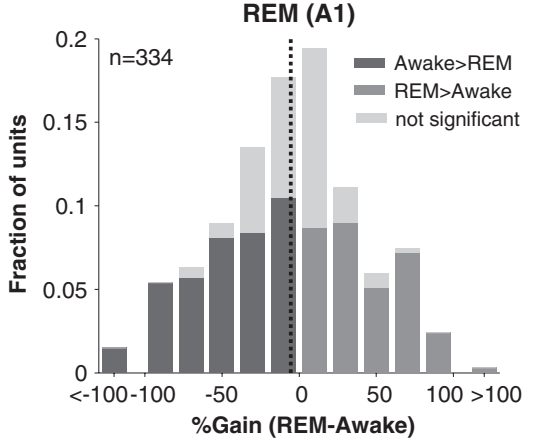

E

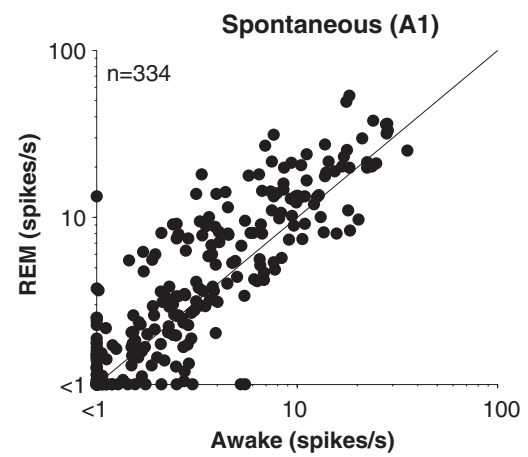

B

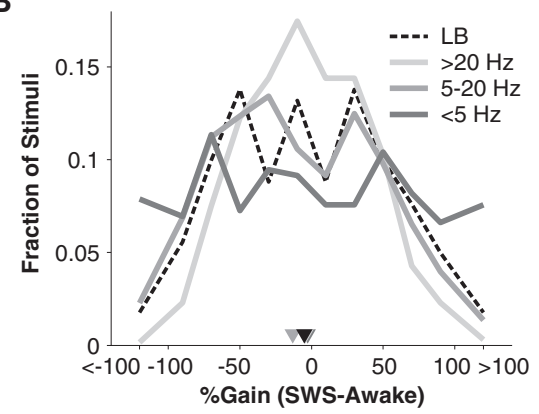

D

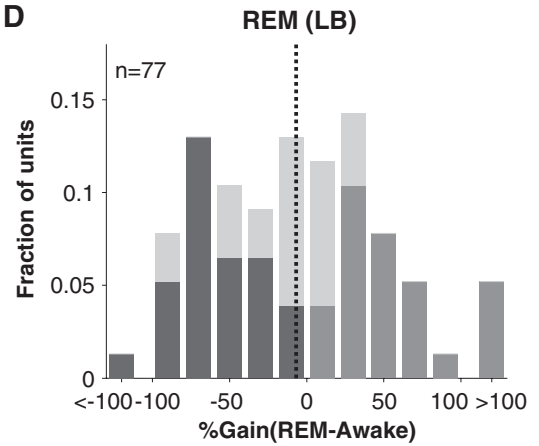

$\mathbf{F}$

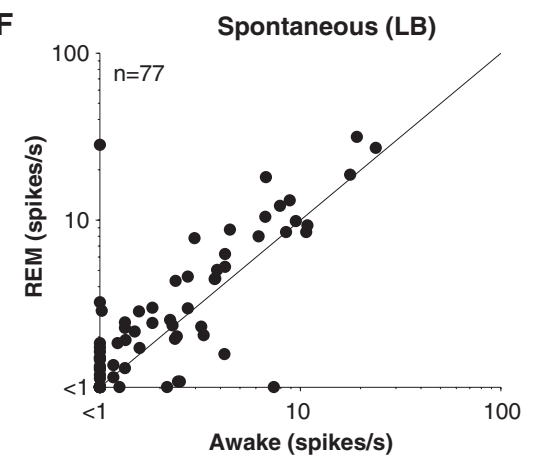

Figure 6. Stimulus-driven and spontaneous activity in LB during sleep. $A$, Histogram of \%Gain (SWS-awake) for cells recorded in $L B(n=84)$. Mean gain $=-8 \%$ (vertical dotted line). Same format as Figure $3 A$. $\boldsymbol{B}$, Control for effects of firing rate in comparing $A 1$ and $L B$. Dividing the $A 1$ data into low $(0-5 \mathrm{~Hz})$, middle $(5-20 \mathrm{~Hz})$, and high $(>20 \mathrm{~Hz})$ firing rates yielded three gain distributions of near-identical medians ( $-3 \%,-13 \%$, and $-4 \%$ respectively) (inverted triangles) but increasing variance $(S D=72 \%, 54 \%, 43 \%)$. The gain distribution was tightest for high firing rates. The distribution for $L B$ (black, dashed line) fell on the $A 1$ distribution for intermediate firing rates $(5-20 \mathrm{~Hz})$ consistent with $L B$ rates averaging $8 \mathrm{~Hz}$. $C$, $\boldsymbol{D}$, Gain histograms in REM for $A 1(C)$ and $L B(D)$. Mean gain $=-5 \%$ (A1) and $-7 \%$ (LB) (vertical dashed lines). $\boldsymbol{E}, \boldsymbol{F}$, Scatter plots of spontaneous firing rates in $\mathrm{A} 1(\boldsymbol{E})$ and $\mathrm{LB}(\boldsymbol{F})$. Although a trend toward higher REM spontaneous rates appears to be present, spontaneous rates were not significantly different from those in awake whether in $\mathrm{A} 1$ or $\mathrm{LB}\left(p_{\mathrm{A} 1}=0.23, p_{\mathrm{LB}}=0.31\right.$, Wilcoxon rank sum, $n_{\mathrm{A} 1}=334, n_{\mathrm{LB}}=$ 77).

(Fig. 5D, inset). Such comparisons between A1 and LB should be qualified by mentioning that different stimuli generally drove LB neurons (e.g., noise and vocalizations). Nonetheless, LB response profiles were not limited to onsets but displayed the same diversity as A1 response profiles.

\section{SWS modulation of $\mathrm{LB}$ responses}

Figure $6 \mathrm{~A}$ shows the effects of SWS on 84 LB units. The distribution is not significantly different from the gain distribution in A1 ( $p=0.80$, Wilcoxon rank sum, $n_{\mathrm{A} 1}=340, n_{\mathrm{LB}}=84$ ). Units were modulated by $40 \%$ in either direction on average, and, over the population, mean spiking rate dropped little in LB during SWS (mean $=-8 \%)$. One concern may be that LB units were not

driven as strongly as A1 units (median driven rate $\mathrm{A} 1=15.9$ and $\mathrm{LB}=9.5$ spikes/s, $p=9.6^{\star} 10^{-8}$, Wilcoxon rank sum, $n_{\mathrm{A} 1}=340, n_{\mathrm{LB}}=84$ ) (Fig. $5 B$ ). Consequently, units in LB may have been tested in a different firing regimen or with nonpreferred stimuli during sleep. As mentioned earlier, however, firing rate and gain were not correlated in A1 (Fig. $3 E)$. Although the gain distribution was tightest for high firing rates and very broad at low firing rates, the mean of the distribution did not vary (Fig. 6B). When the LB gain distribution was overlaid, it fell on the gain distribution for intermediate (5-20 Hz firing rate) firing rates in A1 (Fig. $6 B)$. This was consistent with the majority $(63 \%)$ of $\mathrm{LB}$ rates falling in the $5-20 \mathrm{~Hz}$ range. Therefore, the fact that $\mathrm{LB}$ units were driven to different rates than $\mathrm{A} 1$ units may have marginally increased the amount of heterogeneity we observed in LB but should not affect the conclusion that the mean gain in LB was similar to that in A1 during SWS.

\section{REM modulation of A1 and $\mathrm{LB}$ responses}

Similar to SWS, units in REM underwent both positive and negative modulations (Fig. 6C,D). 33\% of A1 units increased and $40 \%$ decreased their driven response in REM. Mean gain in A1 during REM was only $-5 \%$, representing a small drop of driven activity compared with awake responses (Fig. 6C). A key feature of REM is that spontaneous firing rates were often elevated when the animal entered REM $($ mean awake $=4.0, \mathrm{REM}=5.4 \mathrm{spikes} / \mathrm{s})$. This difference did not reach significance ( $p=0.23$, Wilcoxon rank sum, $n=334$ ) possibly because spontaneous rates were generally very low and near threshold limiting what could be observed extracellularly. However, at higher spontaneous rates, the enhancement in REM compared with wakefulness is more apparent (Fig. $6 E, F)$. No such change in spontaneous rate was visible in SWS (Fig. $3 C$ ).

A general process such as a loss in arousal would have predicted that responses would be depressed and does not seem to explain the bidirectional effects of sleep. Further negating the idea of a simple, general loss of arousal or withdrawal of attention, we found that modulation of responses in REM was independent of modulation in SWS. For example, the unit in Figure 7A had a weak response in SWS but a strong response in REM, as strong as in awake. On a unit-by-unit basis, gains in SWS were poor predictors of gains in REM. For units tested in all 3 states, approximately one-third $(n=135 / 384)$ showed opposite changes in SWS and REM. This is the fraction expected if SWS and REM are uncorrelated (see Materials and Methods). In fact, there was a slight tendency for REM and SWS firing rates to differ more from each other than from awake rates 
[median $\mid$ SWS - REM $\mid=4.6$ and $\mid$ awake $-($ SWS + REM $) / 2 \mid=3.8$ spikes $/$ s, $p=0.008$, Wilcoxon rank sum, $n=384$ ] (Fig. $7 B$ ). The differing modulations in SWS and REM appear to be the result of independent ongoing sleep processes that also have independent effects on external responsiveness.

\section{Controls for awake arousal level}

One concern was that animals may have not reached full awake arousal during night experiments, and awake firing rates could have been underestimated. To address this issue, we compared trials in which the eyes were open versus closed. We also tested awake responses when the animal naturally awakened in a darkened chamber and fell back asleep within minutes as in a typical experiment (natural awakening) versus responses when the experimenter turned the lights on, played loud sounds, and entered the chamber when necessary to actively keep the animal awake (manual awakening). In the eyeopen trials, there was a significant shift of EEG energy into the high frequency bands from the low frequency bands (supplemental Fig. S3A, available at www. jneurosci.org as supplemental material) supporting the notion that these were more aroused conditions than the eyeclosed trials; however, this was not the case when the EEG spectra for manual awakenings were compared with natural awakenings (supplemental Fig. S3A, available at www.jneurosci.org as supplemental material). Perhaps consistent with their EEG spectra not showing a difference, the sleep gains computed using responses from natural or manual awakenings were similar (mean natural awakening $=-4 \%$ vs manual awakening $=$ $-8 \%, p=0.62$, Wilcoxon rank sum, $n=139$ ) (Fig. $8 A$ ). We note here that most manual awakenings were performed at the end to prevent the arousal effect from disrupting sleep or carrying over into the next natural awakening condition. In the second comparison, eye-open trials gave a $9 \%$ improvement in gain compared with eyeclosed trials (mean eyes closed $=-4 \%$ vs eyes open $=-13 \%$ ) suggesting some additional arousal effect that may be related to the shift of EEG power to higher frequencies although this effect did not reach significance ( $p=0.14$, Wilcoxon rank sum, $n=84)$ (Fig. $8 A$ ). So using all awake data regardless of surrogate indicators of arousal level may provide reasonable estimates of gain (mean all awake $=$ $-6 \%$ ) (Fig. $8 A$ ). Finally, we compared the activity in the first five trials to the remaining trials that the animal was awake. Activity immediately on arousal, however, was no greater than in the remaining awake period before the animal fell back asleep ( $p=0.64$, Wilcoxon rank sum, $n=299$ ) (Fig. $8 B$ ). Although animals spent very little time awake, these controls suggested that a sufficient (Fig. $8 \mathrm{~A}$ ) and stable (Fig. $8 B$ ) awake arousal was reached for measuring firing rates without underestimating them.

\section{Stability of neural activity levels throughout sleep}

Overall activity may change over the course of the night because the amplitude of slow waves increases as animals sleep more deeply in the middle of the night. Also, at the end of the night, REM periods become more dominant (Rechtschaffen and Siegel, 2000), or animals may reach lower awake levels. No trend, however, was present in awake or SWS firing rates with sleep episode (Fig. 8C). The relative closeness of SWS and awake responses was consistently present throughout the night. The distributions of maximum unit driven rates early in the night (cycles 1-2) did not differ from those late in the night (cycles $7-10, \sim 5 \mathrm{~h}$ later) whether in the awake state ( $p=0.76$, Wilcoxon rank sum, $n=$ 34 ) or SWS state ( $p=0.53$, Wilcoxon rank sum, $n=34$ ). Correspondingly, mean gain in the early night $(-2 \%)$ was similar to mean gain at the end of the night $(-9 \%)(p=0.97$, Wilcoxon rank sum, $n=34)$. Responses in REM displayed similar consistency (Fig. $8 D$ ). Combined with the reliable recovery of responses within units (Fig. $4 A$ ), these data suggest that statespecific auditory responses were stationary in the population across the duration of the night.

Temporal discharge patterns during awake, SWS, and REM Units in A1 and LB during the awake state often demonstrate sustained firing in addition to an initial onset response (Brugge and Merzenich, 1973; Wang et al., 2005) (also see Fig. 5D). Some anesthetics are known to diminish the later, sustained component (Phillips, 1985). This was not the case in sleep, however. In units whose response started within $60 \mathrm{~ms}$ of stimulus onset, sustained responses were only somewhat more diminished than onset responses (mean gain in SWS, sustained $=-11 \%$ vs onset $=-5 \%, p=0.16$, Wilcoxon rank sum, $n=346$ ), and sus- 
A

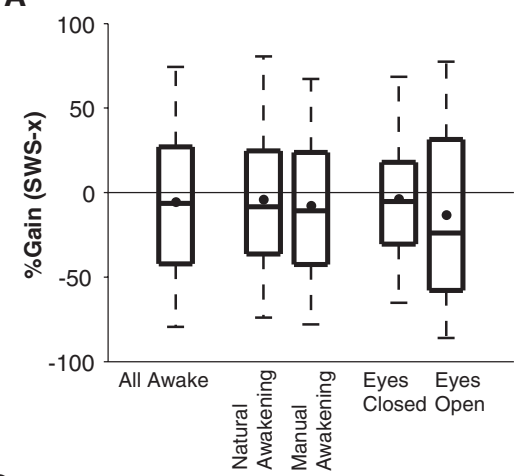

C

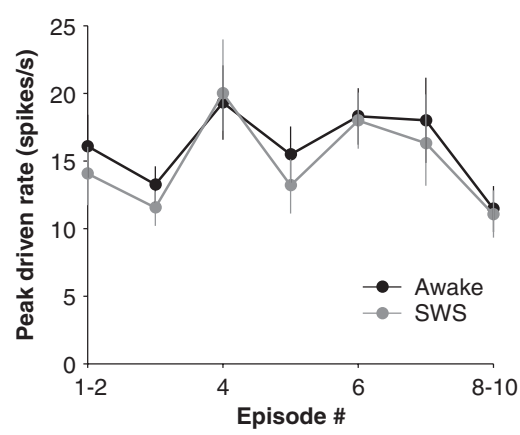

B

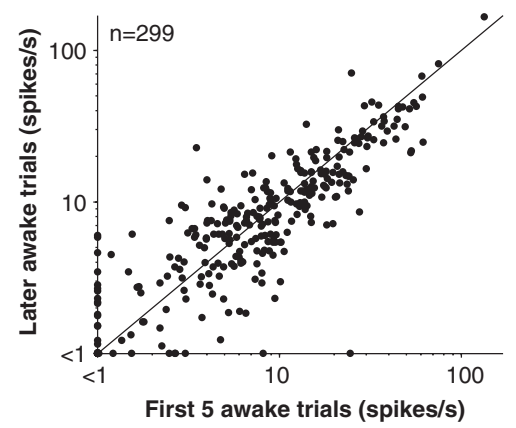

D

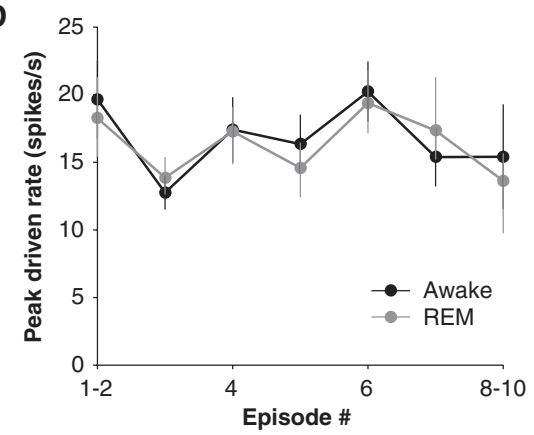

Figure 8. Arousal controls and stability of responses throughout the night. $A$, \%Gain (SWS-awake) recomputed under different arousal conditions. Far left box plot represents SWS gain distribution when all awake conditions were used for comparison $(n=$ 424). Middle box plots compare gains for natural (animal awakens and falls back asleep on its own) versus manual (experimenter induced) awakenings in units tested in both conditions ( $n=139)$. Right bars compare gain for eye-open versus eye-closed trials in units in which at least four trials were collected in both conditions $(n=84)$. Box plots are divided into quartiles, and whiskers represent 5 th to 95 th percentile. $\boldsymbol{B}$, Comparison of awake firing rates for the first five trials after awakening and the remaining trials the animal was awake before falling back asleep. It might be expected that firing would be strongest immediately when the animal awakened, but firing rates did not significantly differ from those before the animal became drowsy again and fell back asleep ( $p=0.64$, Wilcoxon rank sum, $n=299$ ). C, Trend in population activity over the course of the night (error bars represent \pm SEM). Recordings were performed throughout the night starting from when the animal first fell asleep until the early morning. No clear trend in either awake or SWS responses was present, and the two curves follow each other, representing the idiosyncrasies of the population of neurons sampled during each episode. $\boldsymbol{D}$, Same format as in Cexcept for REM responses in comparison to awake responses.

tained responses were still the dominant form of response in sleep (Fig. 9A). Onsets and offsets were a minority in SWS and REM. The tendency for slightly stronger sustained responses in awake led to longer average response windows detected by our algorithm $($ mean awake $=117$, SWS $=102$, REM $=100 \mathrm{~ms}, n=3503$ stimuli). This difference was reflected in the population response histogram (Fig. 9B). The onset is similar in all states, but the awake response diverges slightly in the sustained portion. Still, responses were long-lasting in awake, SWS, and REM.

To further confirm that responses retained their temporal character in SWS and REM, we computed a vector similarity with PSTH patterns in awake. To make this analysis independent of differences in firing rate, only units responsive in both the awake and asleep conditions were considered (a minimal requirement for a PSTH to be constructed), and the correlation measure was normalized by firing rate. Visual examination of the PSTHs of the example cells in Figures 2 and 7 suggests that when there was an appreciable response in one state, its temporal profile tended to mirror that in other states. Over the population, median correlation was $0.78(n=1061)$ between awake and SWS responses and was $0.79(n=708)$ between awake and REM responses. Correlation rarely went below 0.5 (Fig. 9C,D). These correlation values were near the estimated upper limit calculated from the withinstate similarity between odd and even trials (median $=0.73, n=$
2122, for awake/SWS and median $=0.73$, $n=1415$, for awake/REM data used in between-state comparisons). This high similarity suggests that in principle SWS and REM response patterns could support the same temporal code that awake patterns can.

Although mean PSTHs may be similar, their spiking statistics can differ. An important property of SWS responses reported in the literature is their bursting pattern (Edeline et al., 2000; Edeline et al., 2001). Using a simple measure (fraction of spikes occurring within $5 \mathrm{~ms}$ of each other), we found that indeed SWS responses were burstier than awake responses (mean burst fraction in awake $=$ 0.09 vs SWS $=0.11, p=3^{*} 10^{-9}$, Wilcoxon rank sum, $n=1852$ ), and SWS responses were burstier than REM responses (mean REM $=0.09$ vs SWS $=0.11, p=$ $2.1^{*} 10^{-8}$, Wilcoxon rank sum, $n=1852$ ) with no difference between REM and awake ( $p=0.88$, Wilcoxon rank sum, $n=$ 1852). This pattern was true regardless of which minimum ISI criterion $(4,5,8,10$ ms) was used to identify bursts (Fig. 10A).

Stimulus synchronization, the ability of neural responses to follow events in the stimulus, was also preserved in SWS and REM. When tested with sinusoidal amplitude modulated stimuli, units demonstrated similar stimulus synchronization in SWS and awake. This was the case whether measured by the upper limit of stimulus synchronization (mean $F_{\max }$ awake $=14.0$, SWS $=11.6 \mathrm{~Hz}, p=0.37$, Wilcoxon rank sum, $n=58$ ) (Fig. $10 \mathrm{~B}$ ) or by the average vector strength (mean VS awake $=0.45$, SWS $=0.43, p=0.11$, Wilcoxon rank sum, $n=212$ ) (Fig. 10C). In REM, responses to temporally modulated stimuli were also comparable to those in awake (mean $F_{\max }$ awake $=12.7$, REM $=10.2 \mathrm{~Hz}, p=0.46$, Wilcoxon rank sum, $n=40)($ Fig. $10 D)$ (mean VS awake $=0.44, \mathrm{REM}=0.49, p=0.07$, Wilcoxon rank sum, $n=147$ ) (Fig. 10E).

\section{Sleep modulation across stimulus classes}

Up to this point, we have not differentiated between stimuli. Previous behavioral and evoked potential studies have suggested that stimuli are treated differentially in sleep. Behaviorally significant stimuli, for example, tend to be more effective at arousing sleeping subjects (Oswald et al., 1960). Figure 11, $A$ and $B$, summarizes the average gains for the stimulus types that were most often tested in this study (tone, bandpass noise, sAM, vocalizations, and wideband noise). Regardless of stimulus type, mean $\%$ Gain in SWS was usually near $0 \%$ or slightly negative. Within cell comparisons were possible for a few combinations of stimulus types (Fig. 11C,D). Whether for modulated versus unmodulated, narrowband versus broadband, or meaningful (vocalizations) versus nonmeaningful stimuli, no clear difference in gain was seen, and gains averaged near $0 \%$. This suggests that the effects of sleep in the early auditory pathway may generalize across stimulus classes. 
A
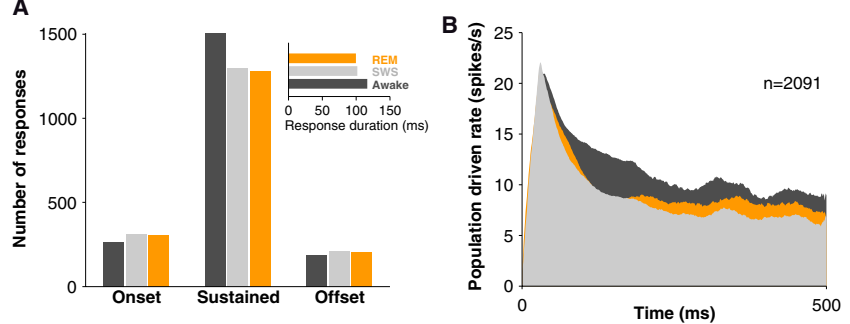

C

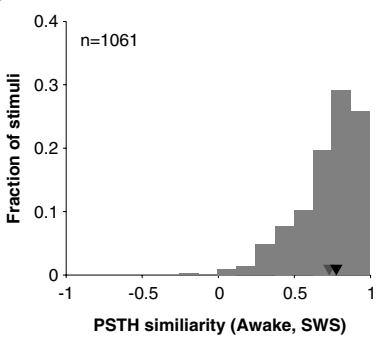

D

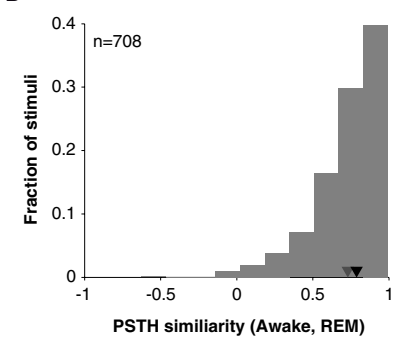

Figure 9. Response patterns observed in sleep. $\boldsymbol{A}$, The number of sustained responses dropped slightly in sleep (SWS or REM) as reflected in the shorter average duration of detected responses (inset). $\boldsymbol{B}$, Population response profiles for all stimuli tested in awake, SWS, and REM. Population PSTH was obtained by averaging, without normalizing, all responses during their respective driven windows. Responses were long-lasting in all three states. $\boldsymbol{C}$, The distribution of similarity values (Pearson's product-moment correlation coefficient) between PSTH profiles in awake and SWS. Response patterns were highly correlated (median $=0.78, n=1061$; black inverted triangle) especially when compared with correlation attainable using odd and even trials from the same state (gray triangle). $\boldsymbol{D}$, The distribution of similarity values between PSTH profiles in awake and REM. Response patterns were highly correlated (median $=0.79, n=$ 708; black inverted triangle).

When we computed the vector similarity of rate profiles across all stimuli (median number of stimuli per curve $=12$ ) between SWS and awake much as we did for PSTH response patterns, median similarity was again quite high $(\rho=0.63, n=353)$ (Fig. $11 E$ ) although not as high as median similarity between awake and SWS PSTHs $(\rho=0.78, n=1061)$. Essentially, a simple multiplicative gain change may account for a substantial (44\%) (Fig. $11 E$ ) amount of the variance between awake and SWS tuning curves and between awake and REM tuning curves (46\%) across all stimuli (Fig. $11 F$ ). These numbers are noteworthy given that the variance explained by using firing rates on odd trials to predict rates on even trials was only $56 \%$ for awake/SWS $(n=$ $704)$ and $61 \%$ for awake/REM $(n=642)$.

\section{Discussion}

In this study, we showed that sleep has bidirectional modulatory effects on neurons in auditory cortex, either raising or lowering firing rates by an average of $40 \%$ compared with wakefulness. Taken as a whole, the population of A1 and LB neurons was quite responsive to sounds during sleep. Data collected while animals naturally went through multiple sleep cycles could be highly nonstationary, but we took great care to ensure that our measurements were stable from cycle to cycle and over the course of the night. Variability in sleep gains was only observed between neurons and not within neurons. An estimate of the effect of the animal's level of awake arousal suggested that awake firing rates were not grossly underestimated $(<10 \%)$.

\section{Comparison to previous studies}

Our results differ from most previous neural studies of sleep in visual and somatosensory cortex (Evarts, 1963; Gücer, 1979; Liv-

ingstone and Hubel, 1981) and even from some studies in the auditory cortex in which neurons were found to be less responsive during sleep (Murata and Kameda, 1963; Brugge and Merzenich, 1973). One issue in comparing across studies is the laminar location of recordings, something that has not been specified in most previous studies. Only in passing did Livingstone and Hubel (1981) note that the deeper cells that they encountered seemed to undergo larger changes in sleep. Layer 4 neurons receive direct thalamic input that is already known to be depressed (Edeline et al., 2000). In upper layers, recurrent intracortical connections between neurons or feedback connections from other areas may re-amplify the response received from input layers (Liu et al., 2007) possibly helping preserve sleep responses. Our recordings tended to be in the upper lamina more so than middle lamina (granular layers) (see Materials and Methods) and may have benefited from any cortical amplification.

We recorded from a New World primate, the common marmoset, which is diurnal. Most previous studies were done in nocturnal animals (cats and guinea pigs). However, we point out that from an evolutionary perspective, sleep seems to have diverged before the split between mammals and birds (Siegel, 2005), and all of the above studies used mammals which display common sleep patterns. Sleep effects may also depend on sensory modality. Each sense has slightly differing subcortical pathways (Kandel et al., 2000). Many nuclei in addition to the thalamus precede auditory cortex whereas the olfactory pathway can bypass the thalamus entirely and go to cortex directly. Future studies comparing two or more modalities in the same experimental animal could help determine the generality of sleep effects.

The variability that we observed in the responses of individual neurons during sleep has been suggested by other studies. Livingstone and Hubel (1981) noted that visual cortical neurons displayed more mixed effects of sleep than those in thalamus. A second group studying auditory thalamus and A1 also observed that cortical neurons (Edeline et al. 2001) were modulated much more heterogeneously compared with thalamic neurons (Edeline et al. 2000).

Human imaging studies in sleeping and lightly sedated patients have found activation in the temporal lobe in response to sounds (Portas et al., 2000; Davis et al., 2007). But another group found a contradictory result (Czisch et al., 2002; Czisch et al., 2004). This issue may be difficult to resolve using imaging because our data suggest that at the much finer neural scale changes during sleep are heterogeneous. In addition to averaging across space, the BOLD signal correlates with synaptic activity more so than spiking activity measured extracellularly (Logothetis et al., 2001; Logothetis, 2008; Viswanathan and Freeman, 2007). One possibility is that imaging signals are dominated by the depressed input coming from thalamus and are not reflecting active cortical processing during sleep.

\section{Potential mechanisms}

In SWS slow oscillations can swing neural excitability between up and down states creating high variability in firing rates (Massimini et al., 2003; Rosanova and Timofeev, 2005). Such an increase in response variability during SWS was absent in our data (Fig. 3D). Although playing loud sounds timed to the occurrence of slow waves can disrupt slow wave activity (Massimini et al., 2003), such a disruption of SWS in the present study seems unlikely given how loudly and how often sounds would have to be played. In our experiments stimuli were presented randomly with respect to EEG rhythms, at long interstimulus intervals, and at a variety of sound levels. The low variance in SWS firing rates 
A

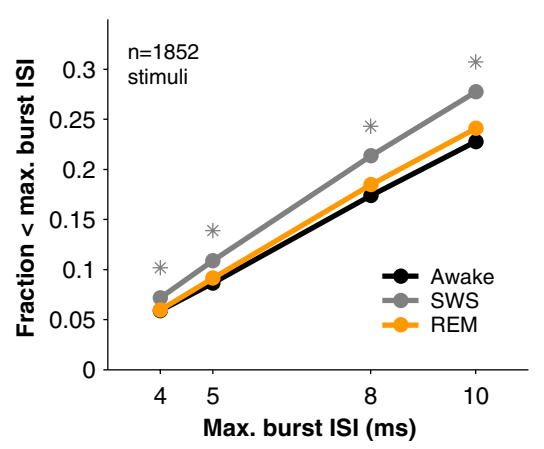

C

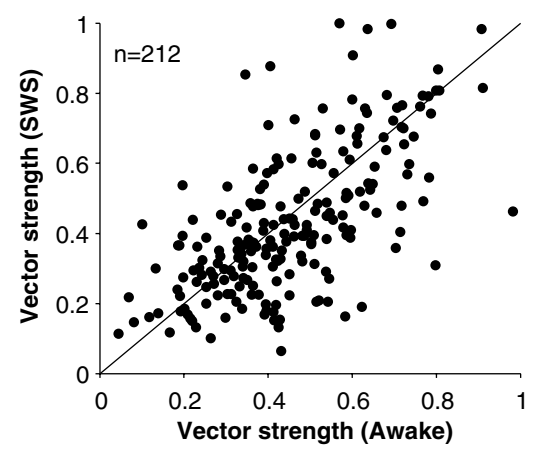

E

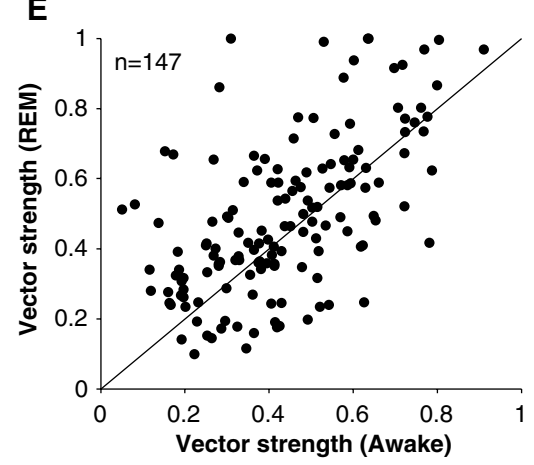

B

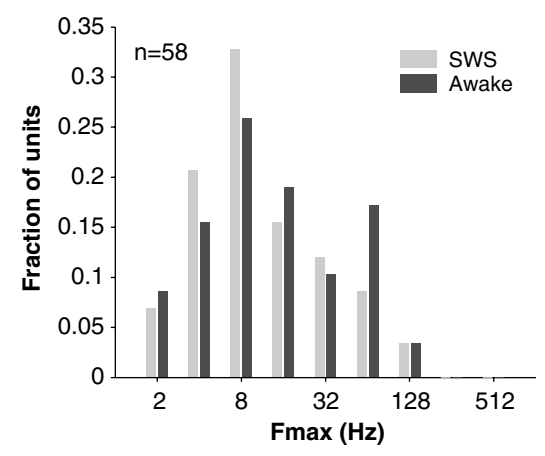

D

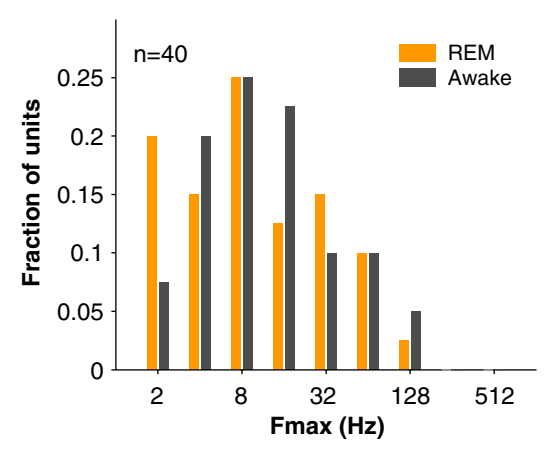

neuron (Logothetis, 2008). Furthermore, internal activations such as replay (Wilson and McNaughton, 1994; Ji and Wilson, 2007) or plasticity phenomena (Jha et al., 2005) could transiently activate subpopulations of neurons and deactivate others during sleep. Recording across multiple nights could resolve this issue because our data cannot speak to stationarity at this longer timescale. The variability of sleep effects may yet be accounted for subcortically as the response properties during sleep in marmoset auditory thalamus are unknown. Finally, there is the possibility that finer gradations of behavioral state influence firing rate. For example, REM can be broken down into tonic and intermittent phasic periods. Future studies could explore the additional effect of variations occurring at a finer timescale than tested here, but we still observed consistent effects by averaging across broadly defined SWS and REM states.

\section{Differentiation from other behavioral state changes}

The observed effects of sleep should be distinguished from those of attention and anesthetics. The strength of attention effects in auditory cortex have been either reported as weak (Benson and Hienz, 1978) or variable (Miller et al., 1972; Hocherman et al., 1976). More recent work has attempted to resolve these issues (Hromádka and Zador, 2007; Fritz et al., 2007). Although individual neurons can have differing attention effects, attention is thought to enhance the overall response of the population in studies of visual cortex (Reynolds and Chelazzi, 2004) unlike the mostly preserved population activity we observed in sleep. Additionally, although attention seems to employ a multiplicative gain (McAdams and Maunsell, 1999) that resembles the nonspecific gains we observed in sleep, we argue that our results could not be accounted for by a withdrawal of attention. For one thing, sleep gains were bidirectional such that almost as many neurons were enhanced as were depressed. Second, gain in REM differed

could be because other factors in addition to slow oscillations (internal activation) control responses to external stimuli. Patterns of stimulus-specific connections could be just as important in setting responses. Indeed, our data were likely a mean picture of activation levels in cortex with the effects of slow oscillations averaged out (Massimini et al., 2003).

Bidirectional modulation was observed across rather than within neurons. Although the source of variability is unknown, we expect that this is not an epiphenomenon but an important window into the network during sleep. It is likely that inhibitory input changes as well as excitatory input which could lead to a myriad of effects depending on how a balance is struck in each from gain in SWS although the animal is not 'attentive' in either condition (explanations involving internally directed attention during dreams could be invoked). Third, an effect of drowsiness was ruled out by post hoc comparisons of trials in which the animal reached different levels of awake arousal.

Although anesthetics may manifest similar cortical oscillations (up and down states) to SWS (Lydic and Baghdoyan, 2005), we found that sustained responses were preserved in sleep unlike under some anesthetics (Brugge and Merzenich, 1973; Phillips, 1985; DeWeese et al., 2003; Hromádka et al., 2008). In the literature, results from anesthetized preparations are often used as an analogy to sleep (Timofeev et al., 1996), but this may not be 

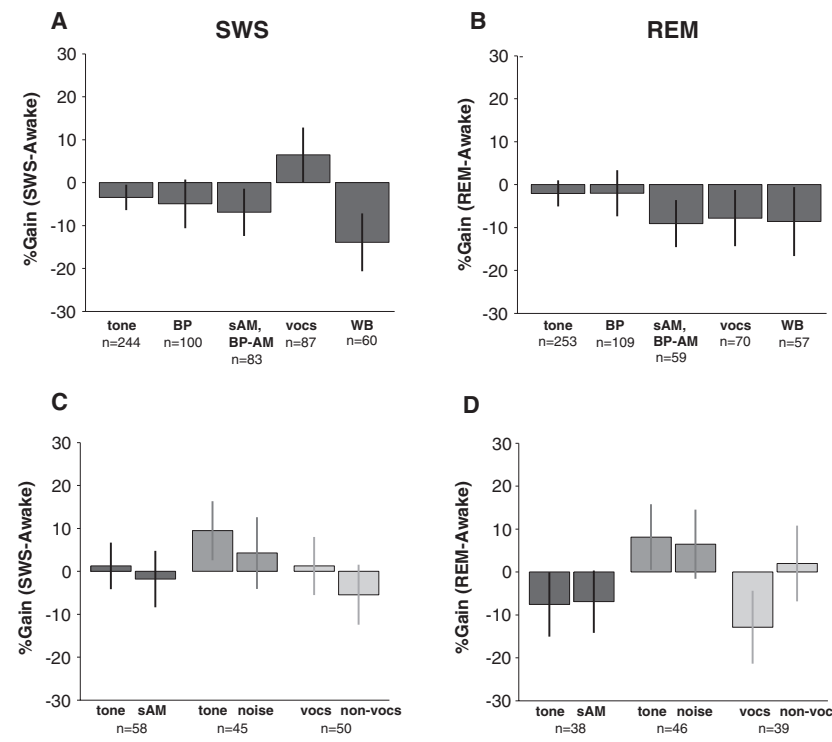

D
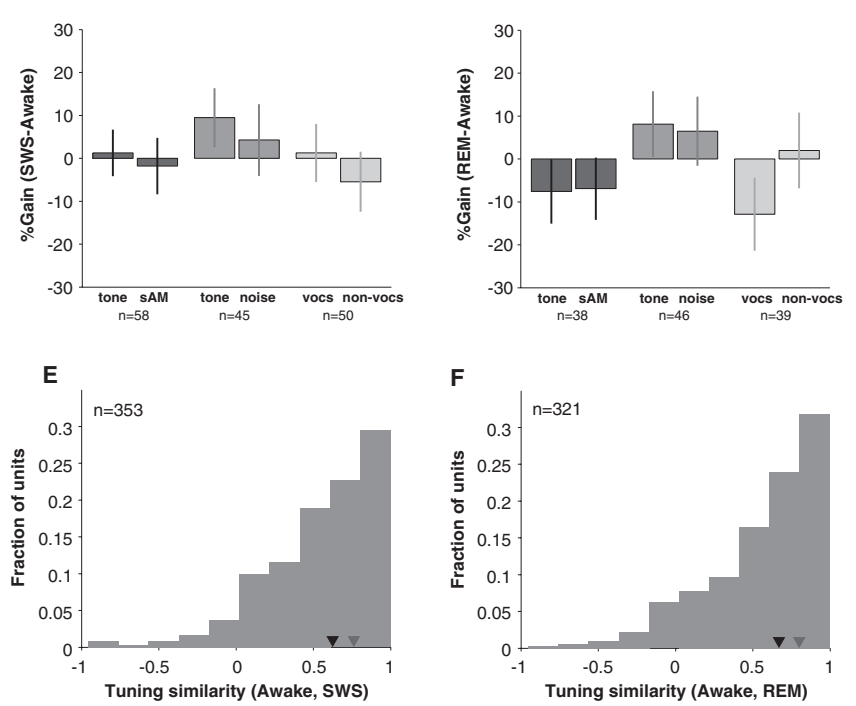

Figure 11. Sleep modulation of all neurons tested across stimulus classes. $\boldsymbol{A}$, We used five main stimulus types to probe neural responses [tones, bandpass noise (BP), sinusoidal amplitude modulated tones (SAM) or noise (BP-SAM), vocalizations (vocs), and wideband noise (WB)]. In general, gains were somewhat negative but not different from $0 \%$ ( $p>0.05$, bootstrap; $n=10,000$ simulations in which awake and SWS firing rates were randomly exchanged) except for wideband noise ( $p=0.05$, bootstrap). $\boldsymbol{B}$, Gains in REM for the five main stimulus types were not different from $0 \%$ ( $p>0.05$, bootstrap). $C$, In many cases, more than one stimulus type was tested on a neuron, and direct comparisons could be made within a neuron. No difference was found in SWS gain for unmodulated versus modulated tones ( $p=0.64, n=$ 58 ), for tones versus narrowband stimuli ( $p=0.47, n=45$ ), and for vocalizations versus nonvocalization stimuli ( $p=0.34, n=50$ ) (Wilcoxon rank sum) (similarly shaded pairs of bars represent data from two different stimulus types collected in the same neuron). Gain remained near $0 \%$ in all cases. $\boldsymbol{D}$, Within-unit comparisons of the effects of REM did not reveal any difference in sleep gains for modulated ( $p=0.95, n=38$ ), larger-bandwidth ( $p=0.98, n=$ 46), or semantically meaningful ( $p=0.22, n=39$ ) (Wilcoxon rank sum) stimuli. $\boldsymbol{E}$, The similarity between awake and SWS firing rate profiles (see Materials and Methods) across all stimuli was relatively high (median $\rho=0.63, n=353$; black inverted triangle) but not as high as within-state similarity (median $=0.76, n=704$; gray inverted triangle). $\boldsymbol{F}$, Awake and REM firing rate profiles across all stimuli also tended to be highly correlated (median $\rho=0.67, n=$ 321; black inverted triangle).

appropriate under conditions of external stimulation. Indeed, the picture we put forward is that sleep responses are more similar to awake responses than to anesthetized responses for external stimulation. The fact that REM behaved independently from SWS suggests the presence of an independent sleep state with no analog under anesthesia. That the sleep state is different from the anesthetized state with regard to single neurons has been seen previously (Torterolo et al., 2002; Cotillon-Williams and Edeline, 2003; Kishikawa et al., 1995; see review by Hennevin et al., 2007).

\section{Functional significance}

As a distance sense audition can serve a protective role through vigilant monitoring of sounds during the night, but it is not at all clear whether and how perception of sounds might take place during sleep. For one thing, our data show an altered distribution of firing rates which could throw off any simple read-out by higher cortical areas such as association areas or frontal cortex (if read-out mechanisms are even online during sleep). Assuming A1 and LB play a role in perception during sleep, our data suggest that information carried by mean activity levels or temporal response patterns may reflect the neural basis for subconscious detection or discrimination of sounds during sleep in humans (Oswald et al., 1960; Beh and Barratt, 1965; Langford et al., 1974; McDonald et al., 1975; Bonnet, 1982; Voss and Harsh, 1998) and animals (Siegel and Langley, 1965; Van Twyver and Garrett, 1972; Halperin and Iorio, 1981; Maho and Hennevin, 1999). The findings reported here stand in contrast to original theories that assumed that the brain shuts off to the external world during sleep (Steriade, 2003a). Given complex modulation across neurons, the idea of a simple gate seems unlikely although it is possible that in higher areas the sleep network is no longer activated externally (Portas et al., 2000; Laureys et al., 2000; Davis et al., 2007) or areas are active but functionally disconnected (Massimini et al., 2005).

\section{References}

Barthó P, Hirase H, Monconduit L, Zugaro M, Harris KD, Buzsáki G (2004) Characterization of neocortical principal cells and interneurons by network interactions and extracellular features. J Neurophysiol 92:600-608.

Bastuji H, Perrin F, Garcia-Larrea L (2002) Semantic analysis of auditory input during sleep: studies with event related potentials. Int J Psychophysiol 46:243-255.

Beh HC, Barratt PE (1965) Discrimination and conditioning during sleep as indicated by the electroencephalogram. Science 147:1470-1471.

Benson DA, Hienz RD (1978) Single-unit activity in the auditory cortex of monkeys selectively attending left vs. right ear stimuli. Brain Res 159:307-320.

Bonnet MH (1982) Performance during sleep. In: Biological rhythms, sleep and performance (Webb WB, ed), pp 205-237. Chichester, UK: Wiley.

Braun AR, Balkin TJ, Wesenten NJ, Carson RE, Varga M, Baldwin P, Selbie S, Belenky G, Herscovitch P (1997) Regional cerebral blood flow throughout the sleep-wake cycle. An H2(15)O PET study. Brain 120:1173-1197.

Brugge JF, Merzenich MM (1973) Responses of neurons in auditory cortex of the macaque monkey to monaural and binaural stimulation. J Neurophysiol 36:1138-1158.

Carskadon MA, Rechtschaffen A (2000) Monitoring and staging human sleep. In: Principles and practices of sleep medicine, Ed 3 (Kryger MH, Roth T, Dement WC, eds), pp 1197-1215. Philadelphia: Saunders.

Coenen AM, Vendrik AJ (1972) Determination of the transfer ratio of cat's geniculate neurons through quasi-intracellular recordings and the relation with the level of alertness. Exp Brain Res 14:227-242.

Cotillon-Williams N, Edeline JM (2003) Evoked oscillations in the thalamo-cortical auditory system are present in anesthetized but not in unanesthetized rats. J Neurophysiol 89:1968-1984.

Crofts HS, Wilson S, Muggleton NG, Nutt DJ, Scott EA, Pearce PC (2001) Investigation of the sleep electrocorticogram of the common marmoset (Callithrix jacchus) using radiotelemetry. Clin Neurophysiol 112:2265-2273.

Czisch M, Wetter TC, Kaufmann C, Pollmächer T, Holsboer F, Auer DP (2002) Altered processing of acoustic stimuli during sleep: reduced auditory activation and visual deactivation detected by a combined fMRI/ EEG study. Neuroimage 16:251-258.

Czisch M, Wehrle R, Kaufmann C, Wetter TC, Holsboer F, Pollmächer T, Auer DP (2004) Functional MRI during sleep: BOLD signal decreases and their electrophysiological correlates. Eur J Neurosci 20:566-574.

Davis MH, Coleman MR, Absalom AR, Rodd JM, Johnsrude IS, Matta BF, Owen AM, Menon DK (2007) Dissociating speech perception and comprehension at reduced levels of awareness. Proc Natl Acad Sci U S A 104:16032-16037. 
Desimone R, Duncan J (1995) Neural mechanisms of selective visual attention. Annu Rev Neurosci 18:193-222.

DeWeese MR, Wehr M, Zador AM (2003) Binary spiking in auditory cortex. J Neurosci 23:7940-7949.

Edeline JM, Manunta Y, Hennevin E (2000) Auditory thalamus neurons during sleep: changes in frequency selectivity, threshold, and receptive field size. J Neurophysiol 84:934-952.

Edeline JM, Dutrieux G, Manunta Y, Hennevin E (2001) Diversity of receptive field changes in auditory cortex during natural sleep. Eur J Neurosci 14:1865-1880.

Efron B (1982) The jackknife, the bootstrap, and other resampling plans. Philadelphia: Society for Industrial and Applied Mathematics.

Evarts EV (1963) Photically evoked responses in visual cortex units during sleep and waking. J Neurophysiol 26:229-248.

Fritz JB, Elhilali M, David SV, Shamma SA (2007) Auditory attentionfocusing the searchlight on sound. Curr Opin Neurobiol 17:437-455.

Goldberg JM, Brown PB (1969) Response of binaural neurons of dog superior olivary complex to dichotic tonal stimuli: some physiological mechanisms of sound localization. J Neurophysiol 32:613-636.

Greenberg DS, Houweling AR, Kerr JN (2008) Population imaging of ongoing neuronal activity in the visual cortex of awake rats. Nat Neurosci 11:749-751.

Gücer G (1979) The effect of sleep upon the transmission of afferent activity in the somatic afferent system. Exp Brain Res 34:287-298.

Halperin JM, Iorio LC (1981) Responsitivity of rats to neutral and dangersignaling stimuli during sleep. Behav Neural Biol 33:213-219.

Hanes DP, Thompson KG, Schall JD (1995) Relationship of presaccadic activity in frontal eye field and supplementary eye field to saccade initiation in macaque: Poisson spike train analysis. Exp Brain Res 103:85-96.

Hennevin E, Huetz C, Edeline JM (2007) Neural representations during sleep: from sensory processing to memory traces. Neurobiol Learn Mem 87:416-440.

Hirsch JC, Fourment A, Marc ME (1983) Sleep-related variations of membrane potential in the lateral geniculate body relay neurons of the cat. Brain Res 259:308-312.

Hocherman S, Benson DA, Goldstein MH Jr, Heffner HE, Hienz RD (1976) Evoked unit activity in auditory cortex of monkeys performing a selective attention task. Brain Res 117:51-68.

Hromádka T, Zador AM (2007) Toward the mechanisms of auditory attention. Hear Res 229:180-185.

Hromádka T, Deweese MR, Zador AM (2008) Sparse representation of sounds in the unanesthetized auditory cortex. PLoS Biol 6:e16.

Hubel DH, Henson CO, Rupert A, Galambos R (1959) “Attention" units in the auditory cortex. Science 129:1279-1280.

Jha SK, Jones BE, Coleman T, Steinmetz N, Law CT, Griffin G, Hawk J, Dabbish N, Kalatsky VA, Frank MG (2005) Sleep-dependent plasticity requires cortical activity. J Neurosci 25:9266-9274.

Ji D, Wilson MA (2007) Coordinated memory replay in the visual cortex and hippocampus during sleep. Nat Neurosci 10:100-107.

Kaas JH, Hackett TA (2000) Subdivisions of auditory cortex and processing streams in primates. Proc Natl Acad Sci U S A 97:11793-11799.

Kandel ER, Schwartz JH, Jessel TM (2000) Principles of neural science. New York: McGraw-Hill.

Kishikawa K, Uchida H, Yamamori Y, Collins JG (1995) Low-threshold neuronal activity of spinal dorsal horn neurons increases during REM sleep in cats: comparison with effects of anesthesia. J Neurophysiol 74:763-769.

Langford GW, Meddis R, Pearson AJ (1974) Awakening latency from sleep for meaningful and nonmeaningful stimuli. Psychophysiology 11:1-5.

Laureys S, Faymonville ME, Degueldre C, Fiore GD, Damas P, Lambermont B, Janssens N, Aerts J, Franck G, Luxen A, Moonen G, Lamy M, Maquet $\mathrm{P}$ (2000) Auditory processing in the vegetative state. Brain 123:1589-1601.

Legéndy CR, Salcman M (1985) Bursts and recurrences of bursts in the spike trains of spontaneously active striate cortex neurons. J Neurophysiol 53:926-939.

Liu BH, Wu GK, Arbuckle R, Tao HW, Zhang LI (2007) Defining cortical frequency tuning with recurrent excitatory circuitry. Nat Neurosci 10:1594-1600.

Livingstone MS, Hubel DH (1981) Effects of sleep and arousal on the processing of visual information in the cat. Nature 291:554-561.
Logothetis NK (2008) What we can do and what we cannot do with fMRI. Nature 453:869-878.

Logothetis NK, Pauls J, Augath M, Trinath T, Oeltermann A (2001) Neurophysiological investigation of the basis of the fMRI signal. Nature 412:150-157.

Lu T, Liang L, Wang X (2001) Temporal and rate representations of timevarying signals in the auditory cortex of awake primates. Nat Neurosci 4:1131-1138.

Lydic R, Baghdoyan HA (2005) Sleep, anesthesiology, and the neurobiology of arousal state control. Anesthesiology 103:1268-1295.

Maho C, Hennevin E (1999) Expression in paradoxical sleep of a conditioned heart rate response. Neuroreport 10:3381-3385.

Mardia KV, Jupp PE (2000) Directional statistics. New York: Wiley.

Mariotti M, Formenti A, Mancia M (1989) Responses of VPL thalamic neurones to peripheral stimulation in wakefulness and sleep. Neurosci Lett 102:70-75.

Massimini M, Rosanova M, Mariotti M (2003) EEG slow ( 1 Hz) waves are associated with nonstationarity of thalamo-cortical sensory processing in the sleeping human. J Neurophysiol 89:1205-1213.

Massimini M, Ferrarelli F, Huber R, Esser SK, Singh H, Tononi G (2005) Breakdown of cortical effective connectivity during sleep. Science 309:2228-2232.

McAdams CJ, Maunsell JH (1999) Effects of attention on orientationtuning functions of single neurons in macaque cortical area V4. J Neurosci 19:431-441.

McDonald DG, Schicht WW, Frazier RE, Shallenberger HD, Edwards DJ (1975) Studies of information processing in sleep. Psychophysiology 12:624-628.

Miller JM, Sutton D, Pfingst B, Ryan A, Beaton R, Gourevitch G (1972) Single cell activity in the auditory cortex of Rhesus monkeys: behavioral dependency. Science 177:449-451.

Mitchell JF, Sundberg KA, Reynolds JH (2007) Differential attentiondependent response modulation across cell classes in macaque visual area V4. Neuron 55:131-141.

Mukhametov LM, Rizzolatti G (1970) The responses of lateral geniculate neurons to flashes of light during the sleep-waking cycle. Arch Ital Biol 108:348-368.

Murata K, Kameda K (1963) The activity of single cortical neurones of unrestrained cats during sleep and wakefulness. Arch Ital Biol 101:306-331.

Oswald I, Taylor AM, Treisman M (1960) Discriminative responses to stimulation during human sleep. Brain 83:440-453.

Peña JL, Pérez-Perera L, Bouvier M, Velluti RA (1999) Sleep and wakefulness modulation of the neuronal firing in the auditory cortex of the guinea pig. Brain Res 816:463-470.

Phillips DP (1985) Temporal response features of cat auditory cortex neurons contributing to sensitivity to tones delivered in the presence of continuous noise. Hear Res 19:253-268.

Portas CM, Krakow K, Allen P, Josephs O, Armony JL, Frith CD (2000) Auditory processing across the sleep-wake cycle: simultaneous EEG and fMRI monitoring in humans. Neuron 28:991-999.

Rauschecker JP, Tian B, Hauser M (1995) Processing of complex sounds in the macaque nonprimary auditory cortex. Science 268:111-114.

Rechtschaffen A, Siegel JM (2000) Sleep and dreaming. In: Principles of neural science, Ed 4 (Kandel ER, Schwartz JH, Jessell TM, eds), pp $936-$ 947. New York: McGraw-Hill.

Reynolds JH, Chelazzi L (2004) Attentional modulation of visual processing. Annu Rev Neurosci 27:611-647.

Rosanova M, Timofeev I (2005) Neuronal mechanisms mediating the variability of somatosensory evoked potentials during sleep oscillations in cats. J Physiol 562:569-582.

Sheinberg DL, Logothetis NK (2001) Noticing familiar objects in real world scenes: the role of temporal cortical neurons in natural vision. J Neurosci 21:1340-1350.

Siegel JM (2005) Clues to the functions of mammalian sleep. Nature 437:1264-1271.

Siegel J, Langley TD (1965) Arousal threshold in the cat as a function of sleep phase and stimulus significance. Experientia 21:740-741.

Steriade M (2003a) The corticothalamic system in sleep. Front Biosci 8:d878-d899.

Steriade M (2003b) Neuronal substrates of sleep and epilepsy. Cambridge, UK: Cambridge UP.

Ter-Mikaelian M, Sanes DH, Semple MN (2007) Transformation of tempo- 
ral properties between auditory midbrain and cortex in the awake Mongolian gerbil. J Neurosci 27:6091-6102.

Timofeev I, Contreras D, Steriade M (1996) Synaptic responsiveness of cortical and thalamic neurones during various phases of slow sleep oscillation in cat. J Physiol 494:265-278.

Torterolo P, Falconi A, Morales-Cobas G, Velluti RA (2002) Inferior colliculus unitary activity in wakefulness, sleep and under barbiturates. Brain Res 935:9-15.

Van Twyver H, Garrett W (1972) Arousal threshold in the rat determined by "meaningful" stimuli. Behav Biol 7:205-215.

Viswanathan A, Freeman RD (2007) Neurometabolic coupling in cerebral cortex reflects synaptic more than spiking activity. Nat Neurosci 10:1308-1312.
Voss U, Harsh J (1998) Information processing and coping style during the wake/sleep transition. J Sleep Res 7:225-232.

Wang X, Lu T, Snider RK, Liang L (2005) Sustained firing in auditory cortex evoked by preferred stimuli. Nature 435:341-346.

Wilson MA, McNaughton BL (1994) Reactivation of hippocampal ensemble memories during sleep. Science 265:676-679.

Wörgötter F, Daunicht WJ, Eckmiller R (1986) An on-line spike form discriminator for extracellular recordings based on an analog correlation technique. J Neurosci Methods 17:141-151.

Zoccolan D, Kouh M, Poggio T, DiCarlo JJ (2007) Trade-off between object selectivity and tolerance in monkey inferotemporal cortex. J Neurosci 27:12292-12307. 